\title{
A study on predicting network corrections in PPP-RTK processing
}

\author{
Kan Wang ${ }^{\mathrm{a}, *}$, Amir Khodabandeh ${ }^{\mathrm{a}}$, Peter Teunissen $^{\mathrm{a}}$ \\ ${ }^{a}$ GNSS Research Centre, Curtin University of Technology, GPO Box U1987, Perth WA 6845, \\ Australia
}

\begin{abstract}
In PPP-RTK processing, the network corrections including the satellite clocks, the satellite phase biases and the ionospheric delays are provided to the users to enable fast single-receiver integer ambiguity resolution. To solve the rank deficiencies in the undifferenced observation equations, the estimable parameters are formed to generate full-rank design matrix. In this contribution, we firstly discuss the interpretation of the estimable parameters without and with a dynamic satellite clock model incorporated in a Kalman filter during the network processing. The functionality of the dynamic satellite clock model is tested in the PPP-RTK processing.

Due to the latency generated by the network processing and data transfer, the network corrections are delayed for the real-time user processing. To bridge the latencies, we discuss and compare two prediction approaches making use of the network corrections without and with the dynamic satellite clock model, respectively. The first prediction approach is based on the polynomial fitting of the estimated network parameters, while the second approach directly follows the dynamic model in the Kalman filter of the network processing and utilises the satellite clock drifts estimated in the network processing. Using $1 \mathrm{~Hz}$ data from two networks in Australia, the influences of the two prediction approaches on the user positioning results are analysed and compared for latencies ranging from 3 to $10 \mathrm{~s}$. The accuracy of the positioning results decreases with the increasing latency of the network products. For a latency of $3 \mathrm{~s}$, the RMS of the horizontal and the vertical coordinates (with respect to the ground truth) do not show large differences applying both prediction approaches. For a latency of $10 \mathrm{~s}$, the prediction approach making use of the satel-

\footnotetext{
${ }^{*}$ Corresponding author

Email addresses: kan.wang@curtin.edu.au (Kan Wang), Amir.Khodabandeh@curtin.edu.au (Amir Khodabandeh), P.Teunissen@curtin.edu.au (Peter Teunissen)
} 
lite clock model has generated slightly better positioning results with the differences of the RMS at mm-level. Further advantages and disadvantages of both prediction approaches are also discussed in this contribution.

Keywords: Satellite clock modelling, Prediction, Latency, PPP-RTK

\section{Introduction}

The Precise Point Positioning (PPP) technique was introduced in the 1990s (Zumberge et al., 1997). By using precise external products for, i.e., the satellite orbits and the satellite clock corrections, and forming linear combinations to eliminate errors like the first-order term of the ionospheric delays, the accuracy of PPP can reach cm-level in the kinematic mode (Yu and Gao, 2017). However, since the phase ambiguities are not resolved to integers, long convergence time from tens of minutes to a couple of hours is needed to reach such accuracy (Banville et al., 2014; Leandro et al., 2011; Yu and Gao, 2017). The real-time kinematic (RTK) technique, in contrast to PPP, solves this problem by generating differential observations. A mm- to cm-level accuracy can be reached within short convergence time after integer ambiguity resolution (IAR), however, only with restricted baseline length of a few tens of kilometres, depending on the ionosphere activity (Janssen and Haasdyk, 2011).

The PPP-RTK technique, which was originally introduced by Wübbena et al. (2005), integrates the advantages of PPP and RTK and enables the integer ambiguity resolution of the PPP. To enable single-receiver integer ambiguity resolution, diverse studies have been performed in the last ten years. The ambiguities can be resolved by providing users the precise satellite-related information like the orbits, the satellite clocks and the satellite phase biases (Mervart et al., 2008; Teunissen et al., 2010; Teunissen and Khodabandeh, 2015). To isolate and estimate undifferenced GPS integer ambiguities, Collins (2008) has also discussed the application of the decoupled clock model by separating the code and the phase clocks, so that the code hardware biases are isolated from the phases and keeps thus the integer property of the ambiguities. Based on the study of Ge et al. (2008), the integer feature of the single-differenced ambiguities can be recovered by applying the single-difference uncalibrated phase delays (UPD), which enables ambiguity resolution. Geng et al. (2010) introduced a method to predict the ionospheric delays, and to accelerate wide-lane and narrow-lane ambiguity resolution. Dau et al. (2003) have discussed the temporal and spatial correlation characteristics of the atmospheric delays and proposed prediction models of the atmospheric delays to resolve the ambiguities of the network stations. In the present contribution we take the undifferenced and uncombined approach (Odijk et al., 2016) to form network-derived estimable satellite 
clocks, satellite phase biases and ionospheric delays, providing them to PPP-RTK users, thereby making single-receiver integer ambiguity resolution feasible.

As mentioned by Odijk et al. (2016), the undifferenced observations without linear combination keeps all parameters in the observation equations and allows a more flexible extension to, e.g., multi-frequency applications. To solve the problem of the resulted rank deficiencies in the undifferenced and uncombined observation equations, however, the estimated parameters need to be formed accordingly to generate a fullrank design matrix with a set of $S$-basis parameters constrained (Teunissen et al., 2010). Applying dynamic models on different sets of parameters may, based on Odijk et al. (2016), change the selection of the $S$-basis parameters and the formulations of the biased parameters. In this study, in addition to the temporal constraints on the ambiguity parameters, the zenith tropospheric delays (ZTDs), the ionospheric delays, the receiver and the satellite hardware biases, a dynamic model for the satellite clock parameters is incorporated in the Kalman filter of the network processing.

During the last twenty years, investigations about latency of the RTK corrections and their influences on the positioning results were performed in diverse studies. According to Weber et al. (2005), the latency of the differential GPS (DGPS) and RTK corrections is typically at the order of less than 3 to $4 \mathrm{~s}$ for communication over internet, wired and wireless phone networks in Germany, and mostly less than $4 \mathrm{~s}$ in Europe. For a limited number of users, the latency in receiving the network information in the network RTK (NRTK) is usually less than $3 \mathrm{~s}$ (El-Mowafy, 2012). To bridge the latency, Vollath et al. (2012), e.g., have used the delta phase approach to estimate the delta position (the rover position increments) by computing its delta range (phase range changes) between the last synchronous epoch with the corrections and the current epoch. The satellite clocks used for computing the range at the current epoch are predicted at the user side with a 2-state Kalman filter assuming a random-walk noise of the satellite clock drift. El-Mowafy and Al-Musawa (2009) have, e.g., proposed a prediction method for the combined measurement corrections of the network stations by employing a level and a trend component for short time period, i.e., several minutes. Qu (2012) performed a study about the effects of different correction data formats, transmission protocols and update frequency of the correction data on the RTK positioning results. In case of a total latency of several seconds, for GPS dual-frequency case, it was found that cm-level accuracy can still be achieved for kinematic solution by using the prediction approach introduced by El-Mowafy and Al-Musawa (2009).

Different from RTK, the PPP-RTK technique uses the State-Space Representation (SSR) (Wübbena et al., 2005) and provides the users individual GNSS-related errors instead of the raw and/or corrected observations (RTCM, 2013). According to 
Leandro et al. (2011), a total correction latency for the Real Time Extended (RTX) system via a satellite link is found to be smaller than $5.6 \mathrm{~s}$ in $99 \%$ of the cases for one test week. In 2011, CNES developed a real-time integer PPP demonstrator with an overall latency for the data collection, processing and transmission estimated between 6 to 8 s (Laurichesse et al., 2010; Laurichesse, 2011). The latency of the products will of course influence the user solutions and as a result, to reduce the induced positioning errors by latency, a reasonable method for prediction of the network corrections is required. In Leandro et al. (2011), e.g., the satellite orbits were estimated with a Kalman filter using numerical integration of the equations of motion, so that the prediction time by the user can last for a few minutes since the last filtering update. Based on the descriptions in RTCM (2013) for the Radio Technical Commission for Maritime Services (RTCM) SSR corrections, polynomial coefficients of the satellite clocks (referred to broadcast satellite clocks) from degrees 0 to 2 can be provided to the users, so that the latency can be bridged by utilising the clock polynomial coefficients. El-Mowafy et al. (2016) has used, e.g., a satellite clock prediction model for the real-time PPP applications that employs these polynomial coefficients and a sinusoid term (Huang et al., 2014) to bridge correction outage from a few minutes to hours, where the contributions of the clock drift rate (the second-order polynomial coefficient) and the sinusoid term are found to be insignificant.

As mentioned by Bröderbauer and Weber (2003), for real-time applications, the satellite clock polynomial coefficients provided by the network could differ in terms of their degrees and the amount of data to fit them. Bröderbauer and Weber (2003) have, e.g., used 48-hour International GNSS Service (IGS) ultra-rapid clock data to estimate the coefficients of a quadratic polynomial and optionally also cyclic terms in a least-squares adjustment for GPS satellite clocks. Wübbena et al. (2005), on the other hand, estimate the satellite clocks dynamically as quadratic polynomial with white noise process in a Kalman filter. Senior et al. (2008) have analysed the stochastic processes of the GPS satellite clocks using the clock products from the IGS with a sampling interval of 5 minutes and $30 \mathrm{~s}$ on the sub-daily regime. Different noise processes were discovered for different generations of GPS satellites, and the white frequency noise (WFN) is found to be dominant in the short-term stochastic behaviours of the GPS IIR/IIR-M satellite clocks.

In this study, a 2-state dynamic satellite clock model is developed for undifferenced and uncombined GPS observations based on the $S$-basis theory. The goal of this contribution is to show the implementation and functionality of this satellite clock model using undifferenced and uncombined observations. For real-time PPPRTK applications, we test and compare two different prediction approaches for the biased terms of the satellite clocks, satellite phase biases and for fast user ambiguity 
resolution, also the slant ionospheric delays (Odijk et al., 2016). The first approach is based on polynomial fits of the network corrections without the dynamic satellite clock model applied in the network Kalman filter. The degrees of the polynomials and the time span to fit the polynomials are studied for different parameters and different prediction intervals in detail. The second approach employs the dynamic model for GPS satellite clocks in the Kalman filter based on van Dierendonck et al. (1984). Together with a general introduction of the processing strategies at the network and the user part, the corresponding $S$-basis parameters and the biased forms of the estimated parameters are studied for cases without and with the satellite clock model in Sections 2 and 3, respectively. In Section 4, the influences of both prediction approaches on the user positioning results are analysed, compared and discussed using $1 \mathrm{~Hz}$ data for 2 networks in Australia with a latency ranging from 3 to $10 \mathrm{~s}$.

\section{Processing and prediction without satellite clock model}

In the network processing, the $f$-frequency observed-minus-computed $(\mathrm{O}-\mathrm{C})$ terms of the undifferenced phase $\left(\Delta \phi_{r, j}^{s}\right)$ and code measurements $\left(\Delta p_{r, j}^{s}\right)$ between the receiver $r(r=1, \cdots, n)$ and the satellite $s(s=1, \cdots, m)$ on frequency $f_{j}(j=$ $1, \cdots, f$ ) can be formulated with (Hofmann-Wellenhof et al., 2008; Kleusberg and Teunissen, 1996):

$$
\begin{aligned}
E\left(\Delta \phi_{r, j}^{s}\left(t_{i}\right)\right)= & g_{r}^{s}\left(t_{i}\right) \tau_{r}\left(t_{i}\right)+d t_{r}\left(t_{i}\right)-d t^{s}\left(t_{i}\right)-\mu_{j} \iota_{r}^{s}\left(t_{i}\right)+\delta_{r, j}\left(t_{i}\right)-\delta_{, j}^{s}\left(t_{i}\right) \\
& +\lambda_{j} z_{r, j}^{s} \\
E\left(\Delta p_{r, j}^{s}\left(t_{i}\right)\right)= & g_{r}^{s}\left(t_{i}\right) \tau_{r}\left(t_{i}\right)+d t_{r}\left(t_{i}\right)-d t^{s}\left(t_{i}\right)+\mu_{j} \iota_{r}^{s}\left(t_{i}\right)+d_{r, j}\left(t_{i}\right)-d_{, j}^{s}\left(t_{i}\right),
\end{aligned}
$$

where $m, n$ and $f$ denote the number of the network stations, the number of the satellites and the number of the frequencies, respectively. $\tau_{r}$ and $g_{r}^{s}$ represent the ZTD parameter and the troposphere mapping function, respectively. The symbols $d t_{r}$ and $d t^{s}$ denote the receiver and the satellite clock parameters in meters, and the first-order term of the slant ionospheric delay on frequency $f_{1}$ is represented by $\iota_{r}^{s}$ with the corresponding ratio $\mu_{j}=\frac{f_{1}^{2}}{f_{j}^{2}}$ in front of it. The phase measurements contain the receiver and the satellite phase biases, denoted by $\delta_{r, j}$ and $\delta_{, j}^{s}$, respectively, and the code measurements contain the terms $d_{r, j}$ and $d_{, j}^{s}$, which denote the receiver and the satellite code biases, respectively. $z_{r, j}^{s}$ expressed in cycles stands for the phase ambiguity parameter, and the wavelength $\lambda_{j}$ converts it into meter-domain. The time point at epoch $i$ is denoted by $t_{i}$, and $\mathrm{E}($.) denotes the expectation operator. As this study is focused on the impact of the predicted satellite clocks, satellite phase biases and ionospheric delays on the user positioning results, the precise station coordinates 
and the precise International GNSS Service (IGS) orbits (Dow et al., 2009; Griffiths and Ray, 2009; IGS orbit, 2017) are assumed to be included in the network O-C terms and are not estimated.

\subsection{Estimability of the parameters}

In Eqs. (1) and (2), rank deficiencies exist between the satellite and the receiver clocks $($ size $=1)$, between the satellite and the receiver hardware biases $($ size $=2 f)$, between the clocks and the hardware biases ( $\operatorname{size}=m+n-1)$, between the ambiguities and the phase hardware biases (size $=f(m+n-1))$ and between the ionospheric delays, the clocks and the hardware biases ( $\operatorname{size}=m+n-1)$. It is necessary to find linearly independent transformations of the parameters with a minimum set of $S$-basis parameters constrained, so that full-rank design matrix can be generated (Teunissen et al., 2010). With the phase ambiguity parameters assumed as constant and the ionospheric delays and the hardware biases estimated as linked parameters in time, to remove the rank deficiencies in the zero-difference observation equations, the O-C terms $\Delta \phi_{r, j}^{s}$ and $\Delta p_{r, j}^{s}$ can be reformulated as:

$$
\begin{aligned}
E\left(\Delta \phi_{r, j}^{s}\left(t_{i}\right)\right)= & g_{r}^{s}\left(t_{i}\right) \tau_{r}\left(t_{i}\right)+d \tilde{t}_{r \neq 1}\left(t_{i}\right)-d \tilde{t}^{s}\left(t_{i}\right)-\mu_{j} \tilde{\iota}_{r}^{s}\left(t_{i}\right)+\tilde{\delta}_{r \neq 1, j}\left(t_{i=1}\right) \\
& +\tilde{\delta}_{r, j}\left(t_{i>1}\right)-\tilde{\delta}_{, j}^{s}\left(t_{i}\right)+\lambda_{j} \tilde{z}_{r \neq 1, j}^{s \neq 1}, \\
E\left(\Delta p_{r, j}^{s}\left(t_{i}\right)\right)= & g_{r}^{s}\left(t_{i}\right) \tau_{r}\left(t_{i}\right)+d \tilde{t}_{r \neq 1}\left(t_{i}\right)-d \tilde{t}^{s}\left(t_{i}\right)+\mu_{j} \tilde{\iota}_{r}^{s}\left(t_{i}\right)+\tilde{d}_{r \neq 1, j>2}\left(t_{i=1}\right) \\
& +\tilde{d}_{r, j}\left(t_{i>1}\right)-\tilde{d}_{, j>2}^{s}\left(t_{i=1}\right)-\tilde{d}_{, j}^{s}\left(t_{i>1}\right),
\end{aligned}
$$

where the biased parameters and the $S$-basis parameters are formulated based on the S-system theory (Baarda, 1981; Teunissen, 1985) and listed in Table 1. The subscript $r=1$ and the superscript $s=1$ represent the reference station and satellite, respectively. (.), IF and (.),GF in Table 1 represent the following formulations:

$$
\begin{aligned}
(.)_{, I F} & =\frac{\mu_{2}(.)_{, 1}-\mu_{1}(.)_{, 2}}{\mu_{2}-\mu_{1}}, \\
(.)_{, G F} & =\frac{(.)_{, 2}-(.)_{, 1}}{\mu_{2}-\mu_{1}} .
\end{aligned}
$$

From Table 1 we see that the estimable satellite clock parameter $d \tilde{t}^{s}$ is a combination of the satellite clock $d t^{s}$, the satellite code bias $d_{, j}^{s}$, the receiver clock $d t_{1}$ and the receiver code bias $d_{1, j}$ of the reference station. Not only the original satellite clocks $d t^{s}$, but also other parameters can influence the temporal behaviour of $d \tilde{t}^{s}$. In case of low-quality receiver clock of the reference station, the temporal behaviour of $d \tilde{t}^{s}$ will be dominated by that of $d t_{1}$. 
Table 1. Estimable parameters and $S$-basis parameters with the ionospheric delays and the hardware biases linked in time. No satellite clock model was applied, see also (Odijk et al., 2016, 2017).

\begin{tabular}{l|l}
\hline Parameter & Formulation \\
\hline$d \tilde{t}_{r \neq 1}\left(t_{i}\right)$ & $d t_{1 r}\left(t_{i}\right)+d_{1 r, I F}\left(t_{1}\right)$ \\
$d \tilde{t}^{s}\left(t_{i}\right)$ & $d t^{s}\left(t_{i}\right)+d_{, I F}^{s}\left(t_{1}\right)-\left(d t_{1}\left(t_{i}\right)+d_{1, I F}\left(t_{1}\right)\right)$ \\
$\tilde{L}_{r}^{s}\left(t_{i}\right)$ & $\iota_{r}^{s}\left(t_{i}\right)+d_{r, G F}\left(t_{1}\right)-d_{, G F}^{s}\left(t_{1}\right)$ \\
$\tilde{\delta}_{r \neq 1, j}\left(t_{i}\right)$ & $\delta_{r, j}\left(t_{i}\right)-\delta_{1, j}\left(t_{1}\right)+\mu_{j} d_{1 r, G F}\left(t_{1}\right)-d_{1 r, I F}\left(t_{1}\right)+\lambda_{j} z_{1 r, j}^{1}$ \\
$\tilde{\delta}_{1, j}\left(t_{i>1}\right)$ & $\delta_{1, j}\left(t_{i}\right)-\delta_{1, j}\left(t_{1}\right)$ \\
$\tilde{\delta}_{, j}^{s}\left(t_{i}\right)$ & $\delta_{, j}^{s}\left(t_{i}\right)+\mu_{j}\left(d_{, G F}^{s}\left(t_{1}\right)-d_{1, G F}\left(t_{1}\right)\right)-\left(d_{, I F}^{s}\left(t_{1}\right)-d_{1, I F}\left(t_{1}\right)\right)$ \\
$\tilde{d}_{r \neq 1, j>2}\left(t_{i}\right)$ & $-\delta_{1, j}\left(t_{1}\right)-\lambda_{j} z_{1, j}^{s}$ \\
$\tilde{d}_{r, j=1,2}\left(t_{i>1}\right)$ & $d_{r, j}\left(t_{i}\right)-d_{1, j}\left(t_{1}\right)-\left(d_{1 r, I F}\left(t_{1}\right)+\mu_{j} d_{1 r, G F}\left(t_{1}\right)\right)$ \\
$\tilde{d}_{1, j>2}\left(t_{i>1}\right)$ & $d_{r, j}\left(t_{i}\right)-d_{r, j}\left(t_{1}\right)$ \\
$\tilde{d}_{, j>2}^{s}\left(t_{i}\right)$ & $d_{1, j}\left(t_{i}\right)-d_{1, j}\left(t_{1}\right)$ \\
$\tilde{d}_{, j=1,2}^{s}\left(t_{i>1}\right)$ & $d_{, j}^{s}\left(t_{i}\right)-\left(d_{, I F}^{s}\left(t_{1}\right)+\mu_{j} d_{, G F}^{s}\left(t_{1}\right)\right)-\left(d_{1, j}\left(t_{1}\right)-\left(d_{1, I F}\left(t_{1}\right)+\mu_{j} d_{1, G F}\left(t_{1}\right)\right)\right)$ \\
$\tilde{z}_{r \neq 1, j}^{s \neq 1}\left(t_{i}\right)-d_{, j}^{s}\left(t_{1}\right)$ \\
\hline$S-$ basis & $z_{1 r, j}^{s}-z_{1 r, j}^{1}$ \\
\hline
\end{tabular}


With the rank deficiencies removed from the design matrix, the biased forms of the network parameters (see Table 1) are estimated using the Curtin PPP-RTK Network Software (Odijk et al., 2016, 2017). The flowchart of the Curtin PPP-RTK network platform is shown in Figure 1a. In the network processing, after reading the input and the observation files, the data go through an epoch-wise pre-processing step with the geometry-free single-channel Detection-Identification-Adaptation (DIA) algorithm (Odijk and Verhagen, 2007). Afterwards, the network parameters are processed in a Kalman filter. The vector of the time-updated parameters $\hat{u}_{i \mid i-1}$ and its variance-covariance matrix $Q_{i \mid i-1}$ can be described as:

$$
\hat{u}_{i \mid i-1}=\Phi_{i \mid i-1} \hat{u}_{i-1 \mid i-1}, \quad Q_{i \mid i-1}=\Phi_{i \mid i-1} Q_{i-1 \mid i-1} \Phi_{i \mid i-1}^{T}+S_{u_{i}}
$$

where $\hat{u}_{i-1 \mid i-1}, Q_{i-1 \mid i-1}, \Phi_{i \mid i-1}$ and $S_{u_{i}}$ represent the vector of the corresponding estimated parameters at the epoch $i-1$, their variance-covariance matrix at the epoch $i-1$, their transition matrix from the epoch $i-1$ to $i$ and their noise variancecovariance matrix at the epoch $i$, respectively.

To capture the structure of the observation equations at the epoch $i$, the design matrix can be represented with a batch formulation (Odijk et al., 2016) with the time-updated parameters in Eq. (7). The measurement update of the observation equation and the variance-covariance matrix $Q_{y y}$ can be formulated as:

$$
E\left[\begin{array}{c}
\hat{u}_{i \mid i-1} \\
y_{i}
\end{array}\right]=\left[\begin{array}{c}
F_{i} \\
A_{i}
\end{array}\right] x_{i}, \quad D\left[\begin{array}{c}
\hat{u}_{i \mid i-1} \\
y_{i}
\end{array}\right]=\left[\begin{array}{cc}
Q_{i \mid i-1} & 0 \\
0 & Q_{y y_{i}}
\end{array}\right]
$$

where $y_{i}, A_{i}$ and $x_{i}$ represent the observations, the design matrix and the estimable parameters at epoch $i$, respectively. $F_{i}$ selects the parameters with dynamic models from $x_{i}$, and $Q_{y y_{i}}$ denotes the variance-covariance matrix of the observations at epoch $i$. $\mathrm{D}($.) denotes the dispersion operator.

In this contribution, we deal with dual-frequency observations in GPS-only scenario (GPS L1 and L2). To enable fast PPP-RTK ambiguity resolution, the ionospheric delays need to be interpolated for each user station and provided to the users together with the satellite clocks and the satellite phase biases (Odijk et al., 2016). The least-squares collocation (Moritz, 1978), which considers the spacial correlation of the network and the user stations, and the best linear unbiased prediction (BLUP) model (Goldberger, 1962; Teunissen and Khodabandeh, 2013) are used for interpolation of the user-specific ionospheric delays (Odijk, 2002) (see also Appendix Appendix A). The network corrections including the biased satellite clock estimates $d \hat{\tilde{t}}{ }^{s}$, the biased satellite phase bias estimates $\hat{\tilde{\delta}}_{, j}^{s}$ and the interpolated user-specific biased ionospheric delays $\hat{\tilde{\iota}}_{u}^{s}$ are provided to the user with the O-C terms of the observation 


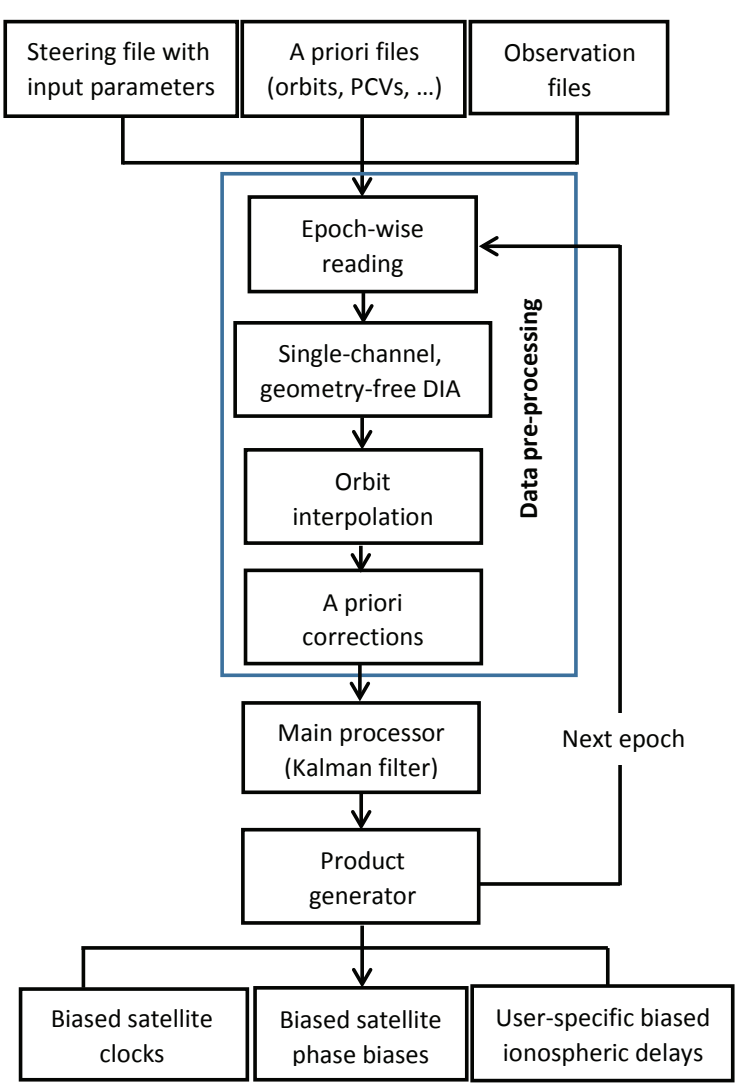

(a) Network platform

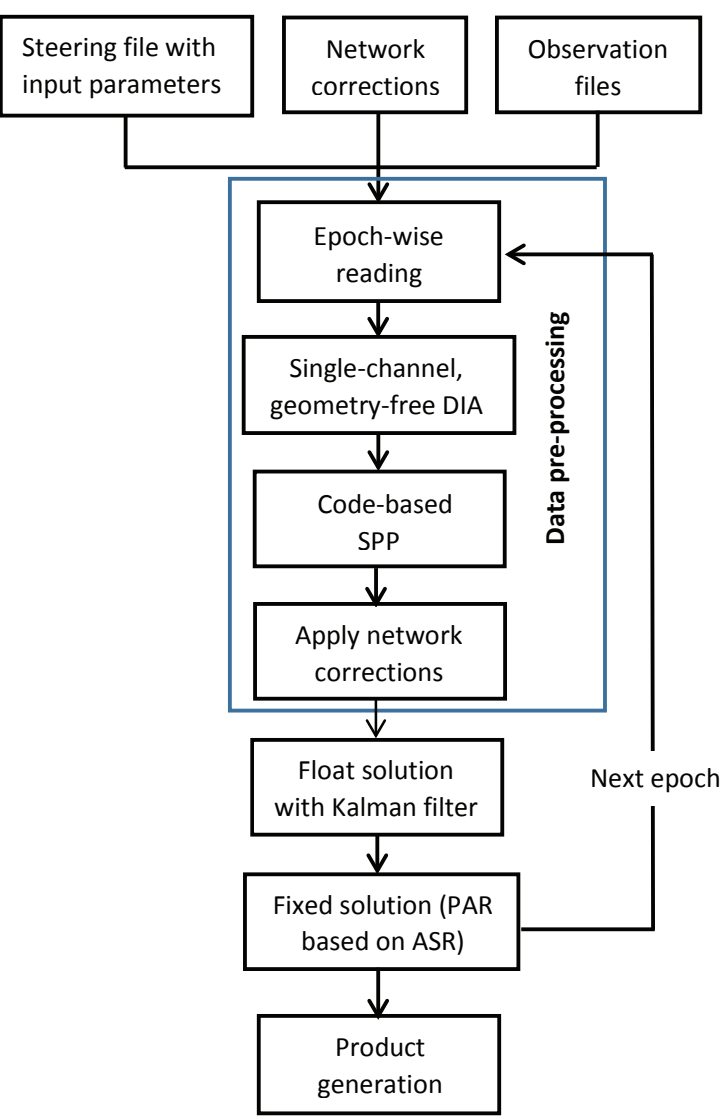

(b) User platform

Fig. 1. Flowcharts of (a) the network and (b) the user platforms of the Curtin PPP-RTK software (Odijk et al., 2017). PCV, DIA, SPP, PAR and ASR are short for Phase Center Variation, Detection-Identification-Adaptation, single point positioning, partial ambiguity resolution and ambiguity success rate, respectively. 
equations formulated as follows:

$$
\begin{aligned}
E\left(\Delta \phi_{u, j}^{s}\left(t_{i}\right)+d \hat{\tilde{\tilde{t}}}^{s}\left(t_{i}\right)+\hat{\tilde{\tilde{\delta}}}_{, j}^{s}\left(t_{i}\right)+\mu_{j} \hat{\tilde{\iota}}_{u}^{s}\left(t_{i}\right)\right)= & \Delta \rho_{u}^{s}\left(t_{i}\right)+d \tilde{t}_{u}\left(t_{i}\right)+\tilde{\delta}_{u, j}\left(t_{i}\right) \\
& +\lambda_{j} \tilde{z}_{u, j}^{s \neq 1}, \\
E\left(\Delta p_{u, j}^{s}\left(t_{i}\right)+d \hat{\tilde{t}}^{s}\left(t_{i}\right)-\mu_{j} \hat{\tilde{\iota}}_{u}^{s}\left(t_{i}\right)\right)= & \Delta \rho_{u}^{s}\left(t_{i}\right)+d \tilde{t}_{u}\left(t_{i}\right)+\tilde{d}_{u, j}\left(t_{i>1}\right) \\
& -\tilde{d}_{, j}^{s}\left(t_{i>1}\right),
\end{aligned}
$$

where the term $\Delta \rho_{u}^{s}$ contains the increment of the user station coordinate and the ZTD in the slant direction. The code-based single point positioning (SPP) (see Fig. 1b) is performed after the single-channel DIA procedure to compute, e.g., the a priori receiver clocks and the a priori receiver coordinates (if not available). After computing the ambiguity-float solutions, the partial ambiguity resolution is performed based on a pre-defined ambiguity success rate (ASR) of $99.99 \%$. The user solutions are saved for both the ambiguity-float and the ambiguity-fixed cases.

For the network and the user processing, two networks in Australia (in Western Australia and Tasmania) with the largest inter-station distance of around 580 and $295 \mathrm{~km}$ (see Fig. 2) were processed from 5:00 to 6:00, 11:00 to 12:00 and 17:00 to 18:00 in GPS Time (GPST) on January 1, 2017 using the Curtin PPP-RTK software (Odijk et al., 2017). Dual-frequency GPS-only $1 \mathrm{~Hz}$ data are used for the processing with an elevation mask of 10 degrees. Since the network is assumed to continuously generate and provide products to users over a long time period, we start the network processing one hour before the user processing. Due to the relatively low accuracy of the regional network corrections for newly risen satellites at low elevation angles, at the user side, the newly risen satellites during this hour with an elevation angle smaller than 12 degrees at the start of the user processing are not used. For newly risen satellites during the user processing, the network corrections of the first 8 minutes have low accuracy due to the filter initialization and are also not used at the user side.

Having partial ambiguity resolution enabled, the absolute errors of the coordinate estimates with respect to the ground truth are computed for all the user stations (in both networks) in all the tested time intervals. The absolute errors are then sorted with their amplitudes for each epoch. The maximal absolute errors that do not exceed $90 \%, 75 \%$ and $50 \%$ of all the sorted absolute errors are used for the plotting in Fig. 3.

Fig. 3 shows the convergence behaviours of the user positioning results with fixed phase ambiguities. The $90 \%, 75 \%$ and $50 \%$ percentiles of the absolute positioning errors with respect to the ground truth provided by the Geoscience Australia (GA, ftp://ftp.ga.gov.au/) are plotted for the first 3 minutes in the North-, East- 


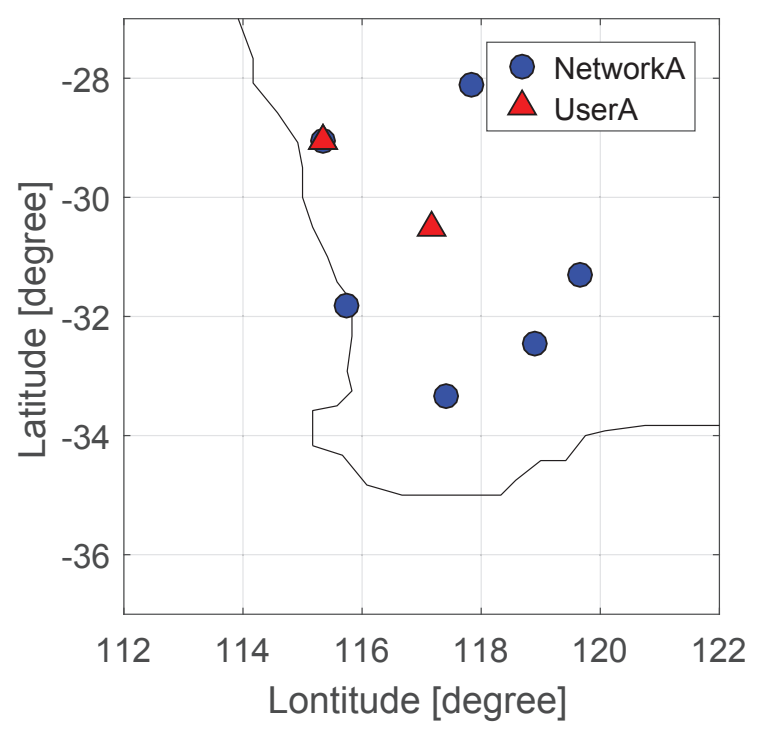

(a) Network A in Western Australia

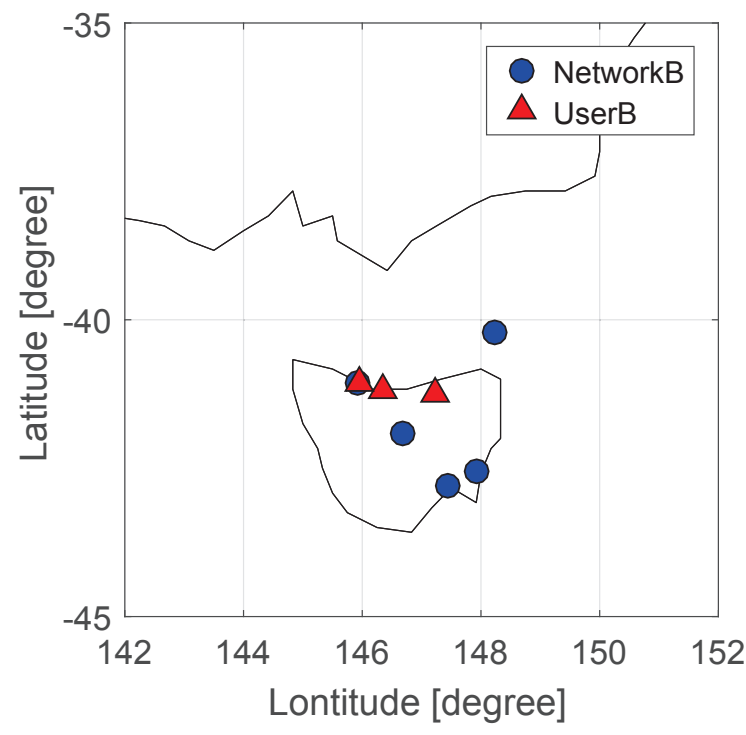

(b) Network B in Tasmania

Fig. 2. Selected network and user stations of (a) the network A (in Western Australia) and (b) the network B (in Tasmania) on January 1, 2017.

and Up-direction. The convergence time is defined as the minimal time required to achieve a certain positioning accuracy for the rest of the processing time.

For the North- and the East-coordinates with fixed ambiguities, it takes 10 and $7 \mathrm{~s}$ to let $90 \%$ of the absolute positioning errors converge to less than $1 \mathrm{dm}$, respectively. $75 \%$ of the absolute positioning errors get less than $5 \mathrm{~cm}$ within $9 \mathrm{~s}$ in the horizontal directions, and the 50\% percentile curves (see the green lines in Figures 3a and 3b), which correspond to the median of the absolute positioning errors, need only $5 \mathrm{~s}$ to reach such accuracy.

The vertical coordinates are in general less accurate than the horizontal coordinates. For the $75 \%$ percentile curves in the kinematic height estimates (see the red line in Figures 3c), $9 \mathrm{~s}$ and $17 \mathrm{~s}$ are needed for the fixed solutions to converge to below 2 and $1 \mathrm{dm}$, respectively.

It is important to remark that although the data are processed in post-processing mode, the data processing is set to mimic the real-time scenario. As shown in Figure 1, Kalman filter is employed for recursive estimation of the parameters, while the recursive DIA (Teunissen, 1990) is used to detect/identify mis-modelled effects and adapt the model accordingly. 


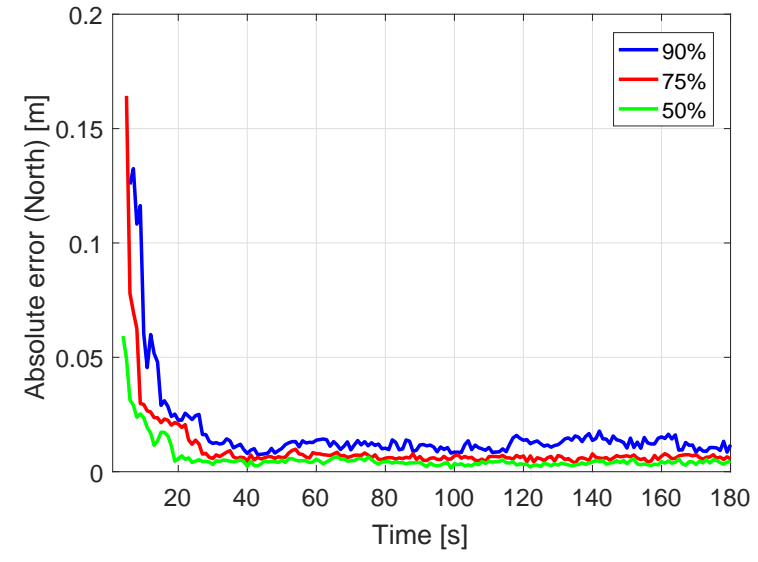

(a) North

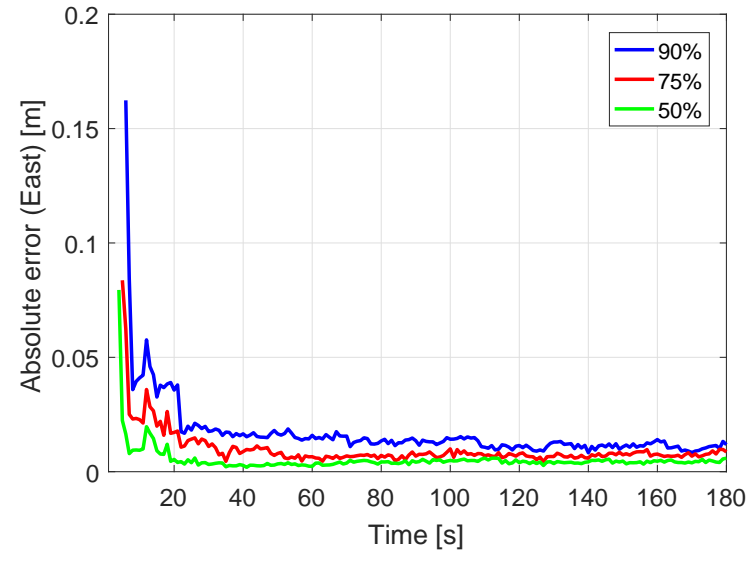

(b) East

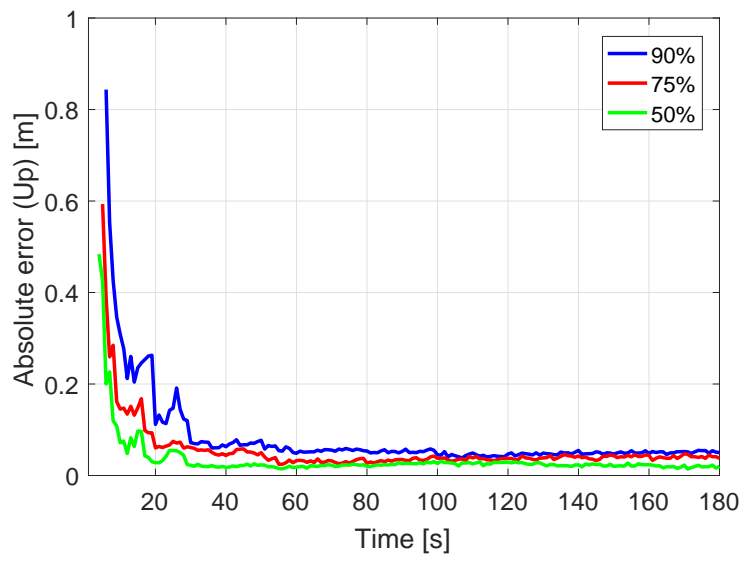

(c) Up

Fig. 3. Convergence behaviours of the (a) North-, (b) East- and (c) Up-coordinates with fixed ambiguities using all user stations from both networks and for all tested time intervals. The $90 \%$ (blue lines), $75 \%$ (red lines) and 50\% (green lines) percentiles of the absolute positioning errors with respect to the ground truth are plotted using $1 \mathrm{~Hz}$ data for the networks $\mathrm{A}$ and $\mathrm{B}$ from 5:00 to 6:00, 11:00 to 12:00 and 17:00 to 18:00 (in GPST) on January 1, 2017. 


\subsection{Approach 1: Prediction without satellite clock model}

Due to the latency of the network products, prediction has to be performed to bridge the time gap between the time point of the user positioning and that of the delayed products. In this study, the network corrections are predicted with a latency ranging from 3 to $10 \mathrm{~s}$.

In the first approach, also called Approach 1, the prediction of the network corrections is based on the estimated satellite clocks $d \tilde{\tilde{t}}^{s}$, the estimated satellite phase biases $\hat{\tilde{\delta}}_{, j}^{s}$ and the interpolated ionospheric delays $\hat{\tilde{\iota}}_{u}^{s}$ (see Section 2.1). For the prediction, the satellite clocks $d \tilde{t}^{s}$ are modelled here as polynomials (in time) with degrees 1 and 2:

- P1: Linear polynomial,

- P2: Quadratic polynomial.

At the same time, the satellite phase biases $\tilde{\delta}_{, j}^{s}$ are modelled either as a constant or a linear polynomial in time:

- B0: Constant,

- B1: Linear polynomial.

The prediction of the user-specific ionospheric delays $\tilde{\iota}_{u}^{s}$ is performed separately for each user station and each satellite and will be introduced later. As the first step, we introduce the prediction strategy for the satellite clocks and the satellite phase biases.

The polynomial coefficients of the biased satellite clocks $d \tilde{t}^{s}$ and the biased satellite phase biases $\tilde{\delta}_{, j}^{s}$ are estimated together:

$$
\begin{aligned}
E\left(d \tilde{t}^{s}\left(t_{i}\right)\right) & =a_{v}^{s}\left(t_{i}-t_{0}\right)^{v}+\cdots+a_{1}^{s}\left(t_{i}-t_{0}\right)+a_{0}^{s} \\
E\left(\tilde{\delta}_{, j}^{s}\left(t_{i}\right)\right) & =b_{p, j}^{s}\left(t_{i}-t_{0}\right)^{p}+\cdots+b_{0, j}^{s}
\end{aligned}
$$

where $a_{k}^{s}(k=0, \cdots, v$ with $v=1$ or 2$)$ and $b_{k, j}^{s}(k=0, \cdots, p$ with $p=0$ or 1$)$ stand for the polynomial coefficients for the satellite clock and the satellite phase bias on frequency $j$, respectively. Assuming that $t_{N}$ and $t_{E}$ represent the time point of the network products and the time interval for estimating the polynomial coefficients, respectively, $t_{0}$ equals to $t_{N}-t_{E}$, and $t_{i}$ varies from $t_{N}-t_{E}$ to $t_{N}$. The biased satellite clocks $d \tilde{t}^{s}$ and satellite phase biases $\tilde{\delta}_{, j}^{s}$ can then be predicted for the time point $t_{N}+t_{P}$ with the estimated polynomial coefficients. 
In Approach 1, the estimation interval $t_{E}$ and the model combination could vary for different prediction intervals $t_{P}$. Since the true values of the biased satellite clocks $d \tilde{t}^{s}$ and biased satellite phase biases $\tilde{\delta}_{, j}^{s}$ are unknown, we take the estimated satellite clocks $d \hat{\tilde{t^{s}}}$ and satellite phase biases $\tilde{\tilde{\delta}}_{, j}^{s}$ at the time point $t_{N}+t_{P}$ as the references to compute the predictions errors. Since the users can eliminate the receiver-related errors by generating between-satellite single-differences of the observations (Khodabandeh and Teunissen, 2015), the prediction errors $\tilde{\omega}_{, j}^{1 s}$ are computed at the betweensatellite level, as the network corrections are effective at the between-satellite level for the user positioning:

$$
\tilde{\omega}_{, j}^{1 s}=d \check{\tilde{t}}^{1 s}+\check{\tilde{\delta}}_{, j}^{1 s}-\left(d \hat{\tilde{t}}^{1 s}+\hat{\tilde{\delta}}_{, j}^{1 s}\right),
$$

where $d \check{\tilde{t}}{ }^{1 s}$ and $\check{\tilde{\delta}}_{, j}^{1 s}$ represent the predicted satellite clocks and satellite phase biases on the between-satellite level at the time point $t_{N}+t_{P}$ using the polynomial coefficients estimated with Eqs. (11) and (12), respectively.

The overall RMS of the prediction errors for the satellite clocks and the satellite phase biases is then calculated as:

$$
\sigma_{\tilde{\omega}}=\sqrt{\frac{\sum_{k=1}^{K} \sum_{j=1}^{h} \sum_{i=1}^{q_{k, j}} \tilde{\omega}\left(t_{i}\right)^{2}}{\sum_{k=1}^{K} \sum_{j=1}^{h} q_{k, j}}},
$$

with

$$
\tilde{\omega}\left(t_{i}\right)=\frac{\sum_{j=1}^{f} \sum_{s=2}^{m} \tilde{\omega}_{, j}^{1 s}\left(t_{i}\right)}{f(m-1)},
$$

where $h$ and $K$ represent the number of the tested time intervals and the number of the networks, respectively. $q_{k, j}$ denotes the number of the epochs with predicted corrections for the network $k$ and the test interval $j$. The prediction starts when the user processing starts. Data with gaps or cycle slips from $t_{N}-t_{E}$ to $t_{N}$ are not used for the evaluation.

The analysis is performed for both networks with different combinations of the satellite clock models (P1 and P2) and the satellite phase bias models (B0 and B1). The estimation intervals $t_{E}$ were tested for each model combination from 1 to $39 \mathrm{~s}$. The estimation interval $t_{E}$ that delivers the minimal $\sigma_{\tilde{\omega}}$ (for all the tested $t_{E}$ ) are plotted in Fig. 4a for each $t_{P}$, and the corresponding minimal $\sigma_{\tilde{\omega}}$ are shown in Fig. $4 \mathrm{~b}$. 


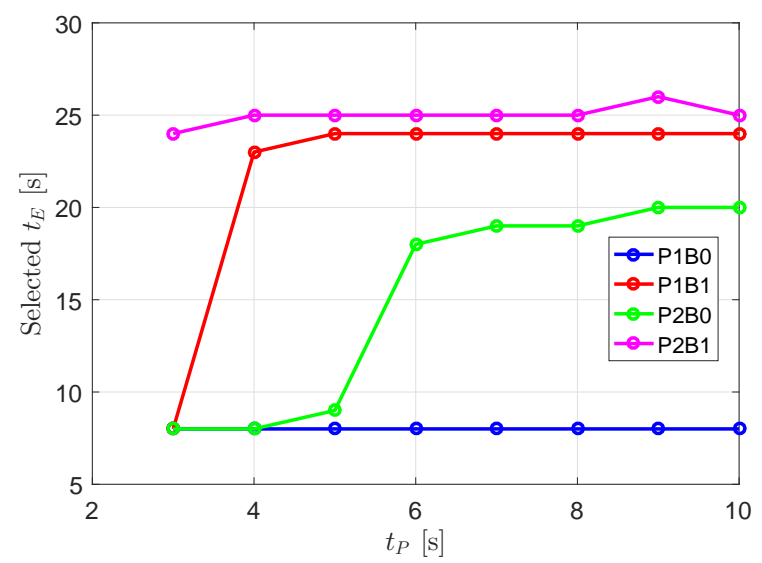

(a) Selected $t_{E}$

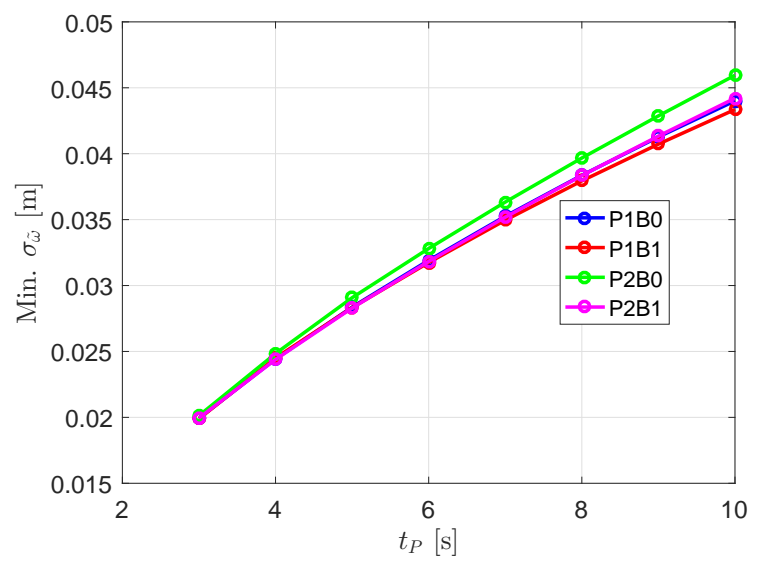

(b) Minimal $\sigma_{\tilde{\omega}}$

Fig. 4. (a) The estimation interval $t_{E}$ that delivers the minimal $\sigma_{\tilde{\omega}}$ (see Eq. (14)) (for all the tested $t_{E}$ from 1 to $39 \mathrm{~s}$ ) and (b) the corresponding minimal $\sigma_{\tilde{\omega}}$ for the predicted satellite clocks and satellite phase biases using different model combinations. P1 and P2 represent polynomials of degrees 1 and 2 for the satellite clocks, and B0 and B1 represent polynomials of degrees 0 and 1 for the satellite phase biases, respectively (see Eqs. (11) and (12)).

From Fig. 4 we see that the minimal $\sigma_{\tilde{\omega}}$ increases with the increasing prediction interval $t_{P}$. The model combination P1B1 (the red line in Fig. 4b) generates lower prediction errors than other model combinations in most cases and will be used for the analysis in Section 4. Fig. 4a also shows that for different prediction time $t_{P}$ the selected $t_{E}$ used for polynomial fitting might be different. In general, a longer $t_{E}$ is required for a longer prediction time $t_{P}$. The selected $t_{E}$ in Fig. 4a are used as the estimation intervals for the corresponding latencies.

A clear disadvantage of Approach 1 is that the post polynomial fitting is not necessarily consistent with the earlier assumptions on the temporal behaviour of the parameters that are placed in the network filter. For instance, if the clock parameters are assumed unlinked in time in the filter, one would violate such assumption by fitting polynomials to the time series of the clock solutions.

The ionospheric delays $\tilde{\iota}^{s}$ are modelled as a linear or a quadratic polynomial in time for each user station and each satellite:

- I1: Linear polynomial

- I2: Quadratic polynomial.

The polynomial coefficients for prediction of the $\tilde{\iota}_{u}^{s}$ are represented by $\alpha_{k}^{s}(k=$ 
$0, \cdots, g$ with $g=1$ or 2 ) and are determined with:

$$
E\left(\tilde{\iota}_{u}^{s}\left(t_{i}\right)\right)=\alpha_{g}^{s}\left(t_{i}-t_{0}\right)^{g}+\cdots+\alpha_{1}^{s}\left(t_{i}-t_{0}\right)+\alpha_{0}^{s}
$$

where $t_{i}$ varies from $t_{N}-t_{E}$ to $t_{N}$. The ionospheric delays $\tilde{\iota}_{u}^{s}$ at the time point $t_{N}+t_{P}$ can then be predicted with the estimated polynomial coefficients.

Similar as the satellite clocks and the satellite phase biases, the between-satellite ionospheric prediction error $\tilde{\xi}_{u}^{1 s}$ is formulated as:

$$
\tilde{\xi}_{u}^{1 s}=\check{\tilde{\iota}}_{u}^{1 s}-\hat{\tilde{\iota}}_{u}^{1 s}
$$

where $\check{\iota}_{u}^{1 s}$ represents the predicted ionospheric delays on between-satellite level for the user station $u$. The overall RMS of the ionospheric prediction errors can then be calculated with:

$$
\sigma_{\tilde{\xi}}=\sqrt{\frac{\sum_{u=1}^{U} \sum_{j=1}^{h} \sum_{i=1}^{q_{u, j}} \tilde{\xi}_{u}\left(t_{i}\right)^{2}}{\sum_{u=1}^{U} \sum_{j=1}^{h} q_{u, j}}},
$$

with

$$
\tilde{\xi}_{u}\left(t_{i}\right)=\frac{\sum_{s=2}^{m} \tilde{\xi}_{u}^{1 s}\left(t_{i}\right)}{m-1},
$$

where $U$ represents the number of the user stations for both networks, and $q_{u, j}$ denotes the number of the epochs with predicted ionospheric delays for the user station $u$ and the test time interval $j$.

The selected estimation intervals $t_{E}$ that delivers the minimal $\sigma_{\tilde{\xi}}$ (for all the tested $t_{E}$ from 1 to $39 \mathrm{~s}$ ) and the corresponding minimal $\sigma_{\tilde{\xi}}$ are shown in Fig. 5 with the prediction interval $t_{P}$ ranging from 3 to $10 \mathrm{~s}$. According to Fig. 5b, the linear polynomial is preferred for the prediction of the ionospheric delays and is used for the analysis in Section 4.

\section{Processing and prediction with satellite clock model}

As mentioned in Odijk et al. (2016), the temporal behaviour of the satellite clocks can, e.g., be modelled as random walk process during a satellite pass (Herring et al., 1990; Pratt et al., 2013). In this study, we model the temporal behaviour of the 


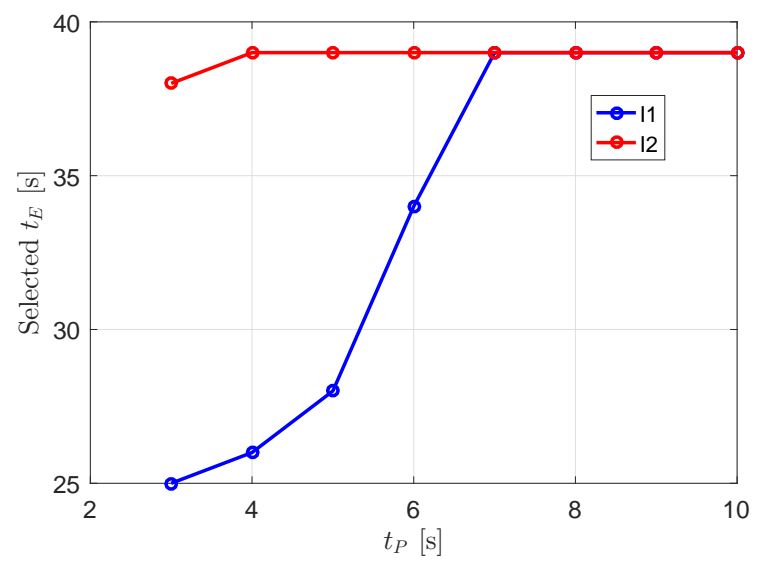

(a) Selected $t_{E}$

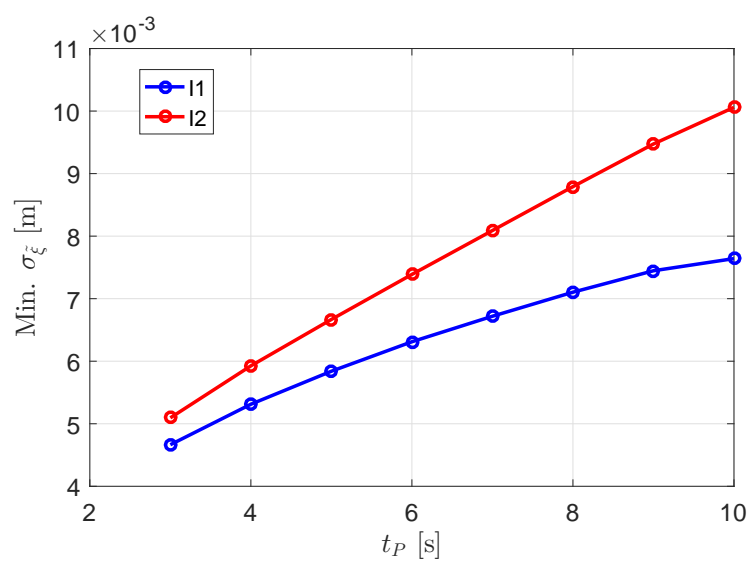

(b) Minimal $\sigma_{\tilde{\xi}}$

Fig. 5. (a) The estimation interval $t_{E}$ that delivers the minimal $\sigma_{\tilde{\xi}}$ (see Eq. (14)) (for all the tested $t_{E}$ from 1 to $39 \mathrm{~s}$ ) and (b) the corresponding minimal $\sigma_{\tilde{\xi}}$ for the predicted ionospheric delays using different models. I1 and I2 represent polynomials of degrees 1 and 2 for the ionospheric delays (see Eq. (16)).

original satellite clocks $d t^{s}$ with the help of a drift parameter $\dot{d} t^{s}$ (Krawinkel and Schön, 2016):

$$
\left[\begin{array}{l}
d t^{s}\left(t_{i}\right) \\
\dot{d} t^{s}\left(t_{i}\right)
\end{array}\right]=\Phi_{i \mid i-1}^{s}\left[\begin{array}{c}
d t^{s}\left(t_{i-1}\right) \\
\dot{d} t^{s}\left(t_{i-1}\right)
\end{array}\right]+\left[\begin{array}{c}
\epsilon^{s}\left(t_{i}\right) \\
\dot{\epsilon}^{s}\left(t_{i}\right)
\end{array}\right]
$$

with

$$
\Phi_{i \mid i-1}^{s}=\left[\begin{array}{cc}
1 & t_{(i-1) i} \\
0 & 1
\end{array}\right]
$$

where $\epsilon^{s}$ and $\dot{\epsilon}^{s}$ denotes the system noise of the satellite clock and the satellite clock drift on satellite $s$, and $t_{(i-1) i}$ stands for the time interval between the $(i-1)$-th and the $i$-th epoch. As in Greenhall (2001), we take two epochs to initialize the satellite clock drift $\dot{d} t$. The $d t^{s}$ is considered as unlinked parameters at the first two epochs.

In order to properly determine the type of the clock noise, the overlapping Allan deviation (ADEV) $\sigma_{A}$ (Allan, 1987; Riley, 2008) is used for the clock stability analysis:

$$
\sigma_{A}(\tau)=\sqrt{\frac{\sum_{i=1}^{M-2 l}\left(r_{i+2 l}^{s}-2 r_{i+l}^{s}+r_{i}^{s}\right)^{2}}{2 \tau^{2}(M-2 l)}},
$$

with

$$
\tau=l \tau_{0},
$$


Table 2. Slopes of the Allan deviations (ADEVs) for different process noise (Riley, 2008).

\begin{tabular}{c|c}
\hline Type of noise & Slope of ADEV \\
\hline White Phase Noise (WPN) & -1 \\
Flicker Phase Noise (FPN) & -1 \\
White Frequency Noise (WFN) & -0.5 \\
Flicker Frequency Noise (FFN) & 0 \\
Random Walk Frequency Noise (RWFN) & 0.5 \\
Frequency Drift (FD) & 1 \\
\hline
\end{tabular}

where $\tau_{0}$ and $\tau$ represent the sampling interval and the averaging time, respectively, and $M$ and $l$ denote the number of the input clock phase data and the averaging factor, respectively. $r_{i}^{s}$ stands for the input clock at epoch $i$ in seconds. The slopes of the ADEVs are related to the noise types as shown in Table 2 (Riley, 2008).

The satellite clock records with a sampling interval of $30 \mathrm{~s}$, provided by the International GNSS Service (IGS), are used to compute the overlapping ADEVs of the GPS satellite clocks (IGS clock, 2017; Noll, 2010) as shown in Fig. 6. We see that the stabilities and the noise types of the GPS satellite clock estimates differ from each other due to the clock types, the individual clock behaviours and even the processing errors generated by the GNSS processing. Due to the changes of the onboard satellite clock behaviours, changing the settings of the clock noise process according to different satellite types or individual satellites increases the complexity and computational load for real-time usage (Hauschild and Montenbruck, 2009) and is not attempted in this study. Based on the mean slope of the ADEVs of -0.5431, we assume that the main noise type of the GPS satellite clocks is the White Frequency Noise (WFN) (see Table 2). The $\sigma_{A}$ with $\tau$ larger than $28800 \mathrm{~s}$ are not used for calculating the mean slope due to the limited amount of data for generating the corresponding $\sigma_{A}$.

According to van Dierendonck et al. (1984), the 2-state (clock and clock drift) variance-covariance matrix for WFN in Kalman-filter is formulated as:

$$
S_{\epsilon^{s}}\left(t_{i}\right)=\left[\begin{array}{cc}
\frac{h_{0}}{2} t_{(i-1) i} & 0 \\
0 & \frac{h_{0}}{2 t_{(i-1) i}}
\end{array}\right] \cdot c^{2}
$$

with the speed of light denoted by $c$, and the coefficient $h_{0}$ formulated as (Barnes et al., 1971):

$$
h_{0}=\sigma_{A}^{2} \cdot 2 \tau \text {. }
$$




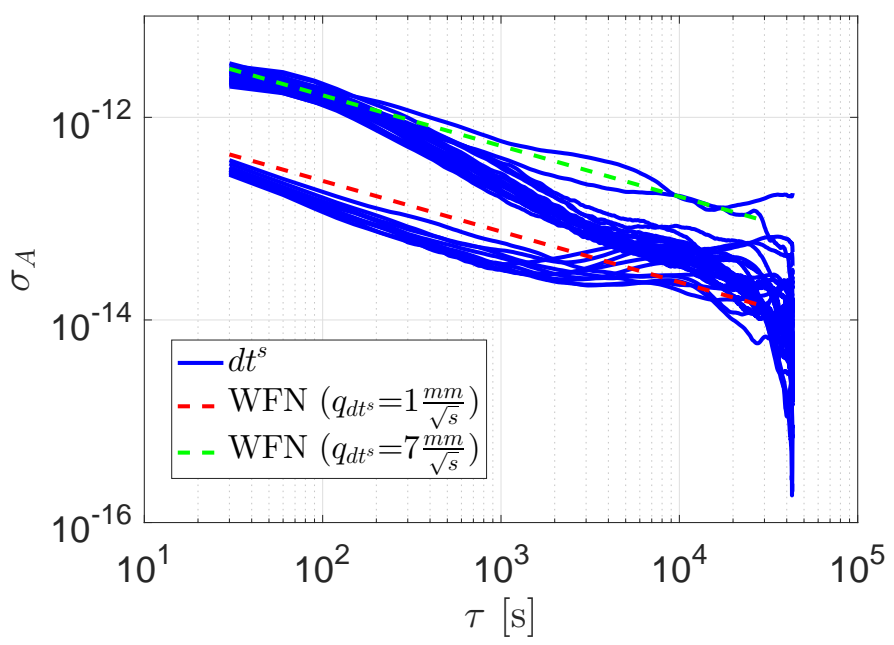

Fig. 6. Overlapping Allan deviations (ADEVs) of the GPS satellite clock estimates on January 1, 2017 with a sampling interval of $30 \mathrm{~s}$. The satellite clock estimates are provided by the International GNSS Service (IGS) (IGS clock, 2017). WFN is short for White Frequency Noise, and $q_{d t^{s}}$ is defined in Eqs. (24), (25) and (26).

In the network processing, the parameter $q_{d t^{s}}=\sqrt{h_{0}} \cdot c$ (with the unit of meter) is given as input parameter. If the ADEV is a straight line with a slope of $-0.5, q_{d t^{s}}$ should be a constant. As a result, the variance matrix $S_{\epsilon^{s}}$ in our satellite clock model is formulated as:

$$
S_{\epsilon^{s}}\left(t_{i}\right)=\left[\begin{array}{ll}
S_{t t} & S_{t v} \\
S_{v t} & S_{v v}
\end{array}\right]=q_{d t^{s}}^{2}\left[\begin{array}{cc}
\frac{t_{(i-1) i}}{2} & 0 \\
0 & \frac{1}{2 t_{(i-1) i}}
\end{array}\right]
$$

Since the behaviours of different satellite clocks differ from each other, and in order not to constrain the satellite clocks too strongly, we have tested the satellite clock model with $q_{d t^{s}}$ varying from 1 to $7 \frac{\mathrm{mm}}{\sqrt{s}}$ (see the red and the green dashed lines in Fig. 6) in Section 4. It should be noted that the possible clock model deficiencies with respect to each satellite clock and the selection of $q_{d t^{s}}$ are sensitive to data with low sampling rate. In this study, we concentrate on the usage of the $1 \mathrm{~Hz}$ data.

\subsection{Estimability of the parameters}

As mentioned in Section 2.1, the biased satellite clock parameter $d \tilde{t}^{s}$ without model contains other terms like the reference receiver clock. When incorporating temporal constraints on the satellite clock parameters, however, the formulation of the biased 
Table 3. Estimable parameters that are changed after imposing the satellite clock model in network processing (see Eq. (29) and Table 1).

\begin{tabular}{l|l}
\hline Parameter & Formulation \\
\hline$d \tilde{\tilde{t}}_{r}\left(t_{i}\right)$ & $d t_{r}\left(t_{i}\right)+d_{1 r, I F}\left(t_{1}\right)-d t_{1}\left(t_{1}\right)-t_{1 i} \dot{d}_{1}\left(t_{2}\right)\left\{\begin{array}{l}r \neq 1, i=1,2 \\
\forall r, i>2\end{array}\right.$ \\
$d \tilde{\tilde{t}}^{s}\left(t_{i}\right)$ & $d t^{s}\left(t_{i}\right)+d_{, I F}^{s}\left(t_{1}\right)-\left(d t_{1}\left(t_{1}\right)+d_{1, I F}\left(t_{1}\right)\right)-t_{1 i} \dot{d} t_{1}\left(t_{2}\right)$ \\
$\dot{d} \tilde{\tilde{t}}^{s}\left(t_{i}\right)$ & $\dot{d}^{s}\left(t_{i}\right)-\dot{d}_{1}\left(t_{2}\right)$
\end{tabular}

satellite clock parameter changes. After applying the satellite clock model, the O-C terms of the phase and the code observations can then be reformulated as:

$$
\begin{aligned}
E\left(\Delta \phi_{r, j}^{s}\left(t_{i}\right)\right)= & g_{r}^{s}\left(t_{i}\right) \tau_{r}\left(t_{i}\right)+d \tilde{\tilde{t}}_{r \neq 1}\left(t_{i=1,2}\right)+d \tilde{\tilde{t}}_{r}\left(t_{i>2}\right)-d \tilde{\tilde{t}}^{s}\left(t_{i}\right)-\mu_{j} \tilde{\tilde{\iota}}_{r}^{s}\left(t_{i}\right) \\
& +\tilde{\tilde{\delta}}_{r \neq 1, j}\left(t_{i=1}\right)+\tilde{\tilde{\delta}}_{r, j}\left(t_{i>1}\right)-\tilde{\tilde{\delta}}_{, j}^{s}\left(t_{i}\right)+\lambda_{j} \tilde{\tilde{z}}_{r \neq 1, j}^{s \neq 1}, \\
E\left(\Delta p_{r, j}^{s}\left(t_{i}\right)\right)= & g_{r}^{s}\left(t_{i}\right) \tau_{r}\left(t_{i}\right)+d \tilde{\tilde{t}}_{r \neq 1}\left(t_{i=1,2}\right)+d \tilde{\tilde{t}}_{r}\left(t_{i>2}\right)-d \tilde{\tilde{t}}^{s}\left(t_{i}\right)+\mu_{j} \tilde{\tilde{\iota}}_{r}^{s}\left(t_{i}\right) \\
& +\tilde{\tilde{d}}_{r \neq 1, j>2}\left(t_{i=1}\right)+\tilde{\tilde{d}}_{r, j}\left(t_{i>1}\right)-\tilde{\tilde{d}}_{, j>2}^{s}\left(t_{i=1}\right)-\tilde{\tilde{d}}_{, j}^{s}\left(t_{i>1}\right),
\end{aligned}
$$

where the changed estimable parameters after applying the satellite clock model are formulated in Table 3. The parameters not listed in Table 3 remain unchanged as listed in Table $1 . d t_{1}\left(t_{i=1,2}\right)$ remain as the $S$-basis parameters, while $d t_{1}\left(t_{i>2}\right)$ are removed. The initial receiver clock drift is defined as:

$$
\dot{d} t_{1}\left(t_{2}\right):=\frac{d t_{1}\left(t_{2}\right)-d t_{1}\left(t_{1}\right)}{t_{12}} .
$$

Based on the formulations in Tables 1 and 3, with the help of the Eq. (20), $d \tilde{\tilde{t}}^{s}$ 
and $\dot{d} \tilde{t}^{s}$ can be formulated with:

$$
\begin{aligned}
d \tilde{\tilde{t}}^{s}\left(t_{i}\right)= & d t^{s}\left(t_{i}\right)+d_{, I F}^{s}\left(t_{1}\right)-\left(d t_{1}\left(t_{1}\right)+d_{1, I F}\left(t_{1}\right)\right)-t_{1 i} \dot{d} t_{1}\left(t_{2}\right) \\
= & d t^{s}\left(t_{i-1}\right)+t_{(i-1) i} \dot{d} t^{s}\left(t_{i-1}\right)+\epsilon^{s}\left(t_{i}\right)+d_{, I F}^{s}\left(t_{1}\right)-\left(d t_{1}\left(t_{1}\right)+d_{1, I F}\left(t_{1}\right)\right) \\
& -\left(t_{1(i-1)}+t_{(i-1) i}\right) \dot{d} t_{1}\left(t_{2}\right) \\
= & d t^{s}\left(t_{i-1}\right)+d_{, I F}^{s}\left(t_{1}\right)-\left(d t_{1}\left(t_{1}\right)+d_{1, I F}\left(t_{1}\right)\right)-t_{1(i-1)} \dot{d} t_{1}\left(t_{2}\right) \\
& +t_{(i-1) i}\left(\dot{d} t^{s}\left(t_{i-1}\right)-\dot{d} t_{1}\left(t_{2}\right)\right)+\epsilon^{s}\left(t_{i}\right) \\
= & d \tilde{\tilde{t}}^{s}\left(t_{i-1}\right)+t_{(i-1) i} \dot{d} \tilde{\tilde{t}}^{s}\left(t_{i-1}\right)+\epsilon^{s}\left(t_{i}\right) \\
\dot{d} \tilde{\tilde{t}}^{s}\left(t_{i}\right)= & \dot{d} t^{s}\left(t_{i}\right)-\dot{d} t_{1}\left(t_{2}\right) \\
= & \dot{d} t^{s}\left(t_{i-1}\right)+\dot{\epsilon}^{s}\left(t_{i}\right)-\dot{d} t_{1}\left(t_{2}\right) \\
= & \dot{d} \tilde{\tilde{t}}^{s}\left(t_{i-1}\right)+\dot{\epsilon}^{s}\left(t_{i}\right) .
\end{aligned}
$$

The same clock model can thus be applied to the new estimable satellite clock and drift:

$$
\left[\begin{array}{c}
d \tilde{\tilde{t}}^{s}\left(t_{i}\right) \\
\dot{d} \tilde{\tilde{t}}^{s}\left(t_{i}\right)
\end{array}\right]=\Phi_{i \mid i-1}^{s}\left[\begin{array}{c}
d \tilde{\tilde{t}}^{s}\left(t_{i-1}\right) \\
\dot{d} \tilde{\tilde{t}}^{s}\left(t_{i-1}\right)
\end{array}\right]+\left[\begin{array}{c}
\epsilon^{s}\left(t_{i}\right) \\
\dot{\epsilon}^{s}\left(t_{i}\right)
\end{array}\right],
$$

\subsection{Approach 2: Prediction with satellite clock model}

As introduced in Section 3.1, the satellite clock $d \tilde{\tilde{t}}^{s}$ and the satellite clock drift $\dot{d} \tilde{\tilde{t}}^{s}$ are estimated at the time point $t_{N}$ applying the dynamic satellite clock model. The estimates $\left(d \hat{\tilde{\tilde{t}}}\right.$ and $d \dot{\tilde{\tilde{t}}}{ }^{s}$ are used to predict the satellite clock $\tilde{\tilde{\tilde{t}}}{ }^{s}$ at the time point $t_{N}+t_{P}:$

$$
d \tilde{\tilde{\tilde{t}}}^{s}\left(t_{N}+t_{P}\right)=d \hat{\tilde{\tilde{t}}}\left(t_{N}\right)+\dot{d \tilde{\tilde{t}}^{s}}\left(t_{N}\right) \cdot t_{P}
$$

If a satellite just newly arises at the time point $t_{i}$, the corresponding satellite clock drift $\dot{d \tilde{\tilde{t}}}{ }^{3}$ is by definition already available at the time point $t_{i+1}$. To avoid large prediction errors due to the inaccurate satellite clock drift at its initialization phase, we wait for $3 \mathrm{~s}$ to let it converge before it is used for the prediction.

In the meanwhile, the predicted $\check{\tilde{\tilde{\delta}}}_{, j}^{s}$ at the time point $t_{N}+t_{P}$ is set to be equal to the estimated $\hat{\tilde{\tilde{\delta}}}_{, j}^{s}$ at the time point $t_{N}$ :

$$
\check{\tilde{\tilde{\delta}}}_{, j}^{s}\left(t_{N}+t_{P}\right)=\hat{\tilde{\tilde{\delta}}}_{, j}^{s}\left(t_{N}\right)
$$


Table 4. Approaches used for the prediction of the network corrections (see Sections 3 and 2).

\begin{tabular}{l|c|c}
\hline Name & Approach & Model \\
\hline 1 & 1 & P1,B1,I1 \\
$2^{-}$ & 2 & $q_{d t^{s}}=7 \frac{m m}{\sqrt{s}}$ \\
2 & 2 & $q_{d t^{s}}=3 \frac{m m}{\sqrt{s}}$ \\
$2^{+}$ & 2 & $q_{d t^{s}}=1 \frac{m m}{\sqrt{s}}$ \\
\hline
\end{tabular}

The estimated ionospheric delays are interpolated for the user station $u$ as described in Section 2.1, denoted as $\tilde{\tilde{\tilde{\iota}}}_{u}^{s}$. The predicted user-specific ionospheric delays $\check{\tilde{u}}_{u}^{s}$ at the time point $t_{N}+t_{P}$ is set to be equal to $\hat{\tilde{\iota}}_{u}^{s}$ at the time point $t_{N}$ :

$$
\check{\tilde{u}}_{u}^{s}\left(t_{N}+t_{P}\right)=\hat{\tilde{\iota}}_{u}^{s}\left(t_{N}\right) \text {. }
$$

\section{Impact of the latency}

As mentioned in Sections 2 and 3, the predicted satellite clocks, satellite phase biases and user-specific ionospheric delays are provided to the users to enable integer ambiguity resolution. Using Approach 1, data gaps shorter than a pre-defined limit within the estimation interval $t_{E}$ are allowed for the predictions. In case of cycle slips from $t_{N}-t_{E}$ to $t_{N}$, only the data after the last cycle slip are used for the prediction of the combined satellite clocks and satellite phase biases. Tests are performed using the GPS dual-frequency $1 \mathrm{~Hz}$ data. For the network and the user processing, the standard deviation of the code and the phase observations are set to be $3 \mathrm{dm}$ and $3 \mathrm{~mm}$ in the zenith direction, respectively. The troposphere ZTDs and the ionospheric delays are temporally constrained with a spectral density of 0.0001 and $0.01 \frac{m}{\sqrt{s}}$, respectively. The receiver and the satellite hardware biases are assumed to be constant. The IGS final orbits were used for both the network and the user processing (IGS orbit, 2017; Noll, 2010).

The user positioning results are analysed for different latencies of the network corrections using different prediction approaches. The approaches used in this section are symbolized as listed in Table 4 . The symbols $2^{-}, 2$ and and $2^{+}$represent the prediction approach using network corrections applying the dynamic satellite clock models with $q_{d t^{s}}$ of 7,3 and $1 \frac{m m}{\sqrt{s}}$, respectively.

As a representative example, the estimated North-, East- and Up-coordinate increments for the user station DPRT using the estimated corrections without latency 

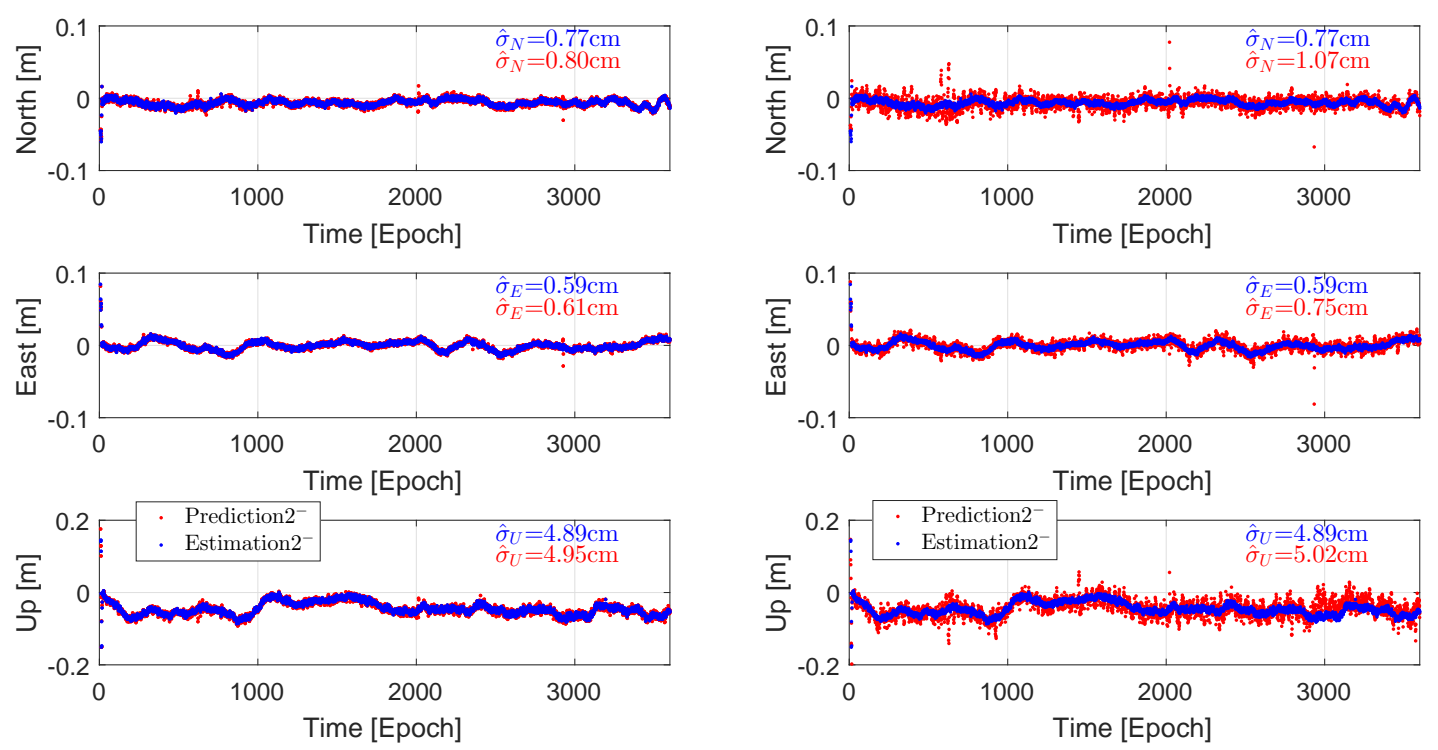

(a) $t_{P}=3 \mathrm{~s}$

(b) $t_{P}=10 \mathrm{~s}$

Fig. 7. Increments of the coordinate estimates of the station DPRT using the estimated network corrections without latency (blue lines) applying the satellite clock model $\left(q_{d t^{s}}=7 \frac{m m}{\sqrt{s}}\right)$ and the predicted network corrections (red lines) with the prediction approach $2^{-}$(see Table 4) from the network B between 17:00 and 18:00 in GPST on January 1, 2017. $1 \mathrm{~Hz}$ data were used for the prediction interval of (a) $3 \mathrm{~s}$ and (b) $10 \mathrm{~s} . \hat{\sigma}_{N}, \hat{\sigma}_{E}$ and $\hat{\sigma}_{U}$ represent the RMS of the estimated increments of the North-, East- and Up-coordinates with respect to the ground truth, respectively, excluding the first 10 epochs as the initialization time for the ambiguity-fixed solutions.

(see the blue lines) and the predicted corrections with a latency of 3 and $10 \mathrm{~s}$ (see the red lines) are shown in Fig. 7. The prediction approach $2^{-}$was applied. The $\hat{\sigma}_{N}$, $\hat{\sigma}_{E}$ and $\hat{\sigma}_{U}$ are the RMS of the coordinate increments excluding the first 10 epochs as the initialization time for the ambiguity-fixed solutions. The limits of the y-axis are scaled to -1 and $1 \mathrm{dm}$ and -2 and $2 \mathrm{dm}$ for the horizontal and the vertical coordinates, respectively.

Fig. 7 shows a clear difference between the user positioning results using the estimated and the predicted network corrections for short and long latencies. In Fig. 7a, for a latency of only $3 \mathrm{~s}$, the horizontal coordinates using the estimated corrections (see the blue lines) agree well with those using the predicted corrections (see the red lines), i.e., with a degradation of the RMS at sub-mm level. In Fig. 7b, with a longer latency of $10 \mathrm{~s}$, the degradation of the RMS is increased to mm-level. 
Around e.g. the 2011-th and the 2924-th epoch, outliers can be observed in the user positioning results in the red dots. This is a joint effect of the latency and the discontinuities of the network corrections. In case of latencies, discontinuities of the network corrections can only be reflected in the predicted corrections a few seconds later. The resulted deviations between the predicted and the estimated network corrections causes the outliers in the user positioning results. In Fig. 7, systematic patterns can be observed in the coordinate estimates. The patterns repeat on the second day and are assumed to be caused by the multipath effects.

To show and compare the impact of the predicted network corrections using different approaches on the user positioning results, the overall RMS of the coordinate increments are computed for all user stations of both networks and all the tested time intervals. The overall RMS of the coordinate increments with respect to the ground truth are computed as follows:

$$
\hat{\bar{\sigma}}_{x}=\sqrt{\frac{\sum_{u=1}^{U} \sum_{j=1}^{h} \sum_{i=v_{0}}^{v_{e}} x\left(t_{i}\right)^{2}}{\sum_{u=1}^{U} \sum_{j=1}^{h}\left(v_{e}-v_{0}+1\right)}},
$$

where $v_{0}$ and $v_{e}$ represent the starting and the ending epochs, and as before, $U$ and $h$ stand for the number of the user stations for both networks and the tested time intervals, respectively. $x\left(t_{i}\right)$ denotes the corresponding coordinate increments with respect to the ground truth. Allowing 10 epochs as the initialization time, the computation starts from $v_{0}$ of the 11-th epoch.

Fig. 8 shows the overall RMS of the user coordinates increments with respect to different latencies using different prediction approaches. $t_{P}$ in the x-axis represents the prediction interval, i.e., the latency. $t_{P}=0 s$ stands for the case without latency. As shown in Fig. 8, the overall RMS of the coordinate increments increases as the latency increases. Among different prediction approaches, we observe that Approach 2 , i.e., the one making use of the dynamic satellite clock model in the Kalman filter of the network processing, has provided slightly better positioning results. For a latency up to $10 \mathrm{~s}$, the RMS of the horizontal coordinates has increased by several millimetres compared to the case without latency. The vertical coordinates are in general less accurate than the horizontal coordinates. The RMS of the vertical coordinates amount to several centimetres at $t_{P}=0 \mathrm{~s}$, i.e., without latency. For a latency up to $10 \mathrm{~s}$, the corresponding RMS are degraded by several millimetres. Using the prediction approach 2, no obvious difference is detected when applying the different satellite clock constraints in our tests within a latency up to $10 \mathrm{~s}$. 

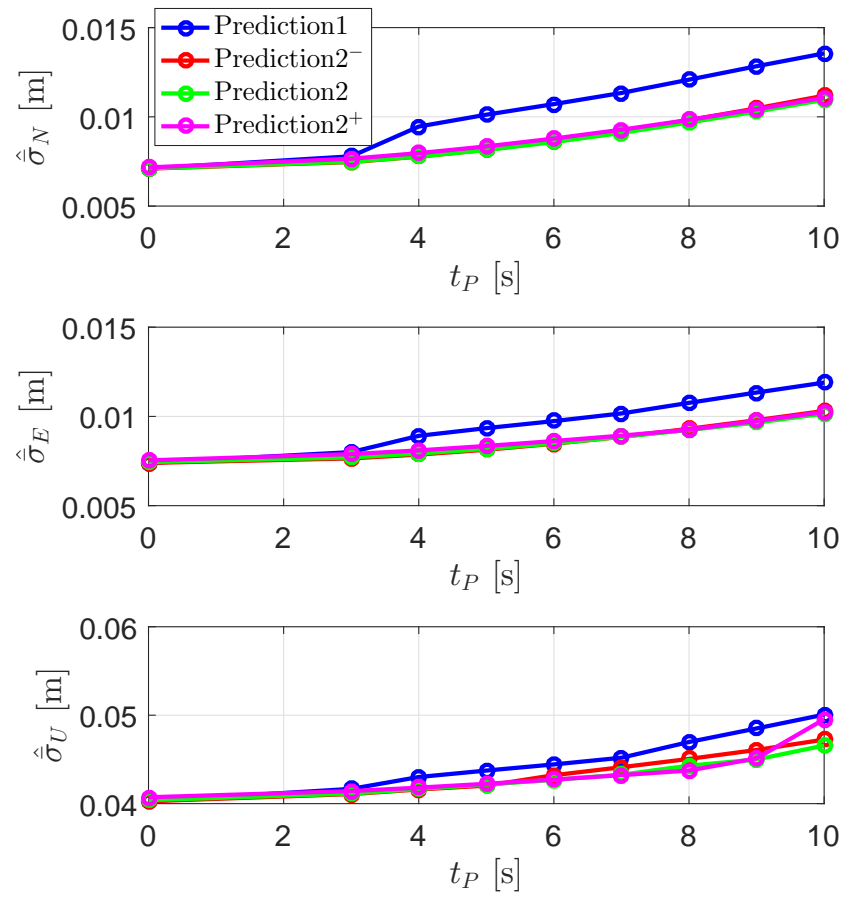

Fig. 8. The overall RMS of the coordinate increments for all user stations from both networks and all tested time intervals (from 5:00 to 6:00, 11:00 to 12:00 and 17:00 to 18:00 in GPST) on January 1, 2017. The approaches used for the prediction of the network corrections are listed in Table 4. 
To further test the functionality of both the approaches on another day, all the user stations in both networks are processed from 11:00 to 12:00 (in GPST) on January 6, 2017. The overall RMS applying Approach $2^{+}$with a latency of $10 \mathrm{~s}$ is, e.g., slightly lower than that applying Approach 1 with a difference of 1.1 and $0.5 \mathrm{~mm}$ in the North- and East-directions, and $1.3 \mathrm{~mm}$ in the vertical direction. We see that the differences in the user positioning results using both approaches are not very significant. However, Approach 1 (by fitting polynomials) is based on the estimated parameters of the network processing. This does not only require additional time for separate computation after the network processing, the selection of the networks, the multipath effects, the satellite geometry and the ionospheric activity may all affect the selection of the models and the estimation time $t_{E}$ for different latencies. Compared to Approach 1, Approach 2 is a more general and efficient approach that directly follows the dynamic models of the network Kalman filter.

\section{Conclusions}

In the PPP-RTK processing, to enable integer ambiguity resolution at the user side, the network corrections including the satellite clocks, satellite phase biases and for fast ambiguity resolution, the ionospheric delays are provided to the users. In practical use, however, the network products are delayed due to the data collection, the network processing and the data transfer. This latency extends to several seconds and could influence the real-time user positioning. This contribution tries to find and compare different solutions with regards to this problem.

To realise the real-time user positioning, predictions of the network corrections cannot be avoided. Based on the characteristics of the temporal behaviour of the GPS satellite clocks, a 2-state dynamic model based on undifferenced and uncombined observations is incorporated into the network processing and thus, enables the prediction of the satellite clocks with the drift parameter estimated in the Kalmanfilter. However, before applying the satellite clock model, we should be very careful with the real contents of the estimated parameters. To solve the rank deficiencies in the observation equations, linearly independent transformations of the parameters, but not the original parameters, were estimated with a minimum set of the $S$-basis parameters constrained. When estimating the satellite clocks as unlinked parameters, e.g., the biased satellite clock parameter contains other terms like the satellite code biases, the receiver code biases and the receiver clocks of the reference station. In this contribution, also based on diverse previous studies, a dynamic satellite clock model is incorporated into the network processing with this issue clarified in detail.

Using the $1 \mathrm{~Hz}$ data from 2 networks in Australia, we predicted the network corrections with two different approaches based on the data without and with the 
satellite clock model, respectively. The tested prediction intervals vary from 3 to $10 \mathrm{~s}$, which correspond to a range of possible latencies of the network corrections. We see that the accuracy of the user coordinate solutions is related to the latency itself. Longer latency, which also requires a longer prediction interval of the network corrections, leads to larger RMS in the user positioning results. The degradation of the RMS of the coordinates is generally at mm-level for a latency up to $10 \mathrm{~s}$. Comparing the two prediction approaches in our tests, we find that Approach 2, namely the one making use of the satellite clock model at the network part, has provided slightly better positioning results. Approach 1 based on polynomial fitting of the estimated parameters does not only require separate computation after the network processing, the polynomial degrees and the amount of the data used for estimating the polynomial coefficients may also vary according to the selection of the network, the measurement environment, the satellite geometry and the ionosphere activity. Compared to Approach 1, Approach 2 directly follows the dynamic models in the Kalman filter and is found to be a more efficient and general approach for prediction of the network corrections in case of latencies.

\section{Acknowledgments}

We would like to thank the IGS and Geoscience Australia (Geoscience Australia 2017) for providing the GNSS products and the $1 \mathrm{~Hz}$ observation data on their servers. We are also grateful for the contributions of our colleagues in the GNSS Research Centre, Curtin University of Technology, in their development of the Curtin PPP-RTK Software.

\section{Appendix A. Interpolation of the ionospheric delays}

For interpolation of the user-specific ionospheric delays, denoted as $\tilde{\iota}_{u}^{s}$, the best linear unbiased prediction (BLUP) method (Teunissen and Khodabandeh 2013) can be used as follows:

$$
E\left(\left[\begin{array}{c}
\tilde{\imath} \\
\tilde{\iota}_{u}
\end{array}\right]\right)=\left[\begin{array}{cc}
C & C_{v} \\
I_{m} & O_{m \times(n-1)}
\end{array}\right]\left[\begin{array}{c}
\bar{\iota}_{o} \\
v
\end{array}\right], \quad W=S^{-1}=\left[\begin{array}{cc}
S_{11} & S_{12} \\
S_{21} & S_{22}
\end{array}\right]^{-1},
$$


with

$$
\begin{aligned}
\hat{\tilde{\iota}} & =\left[\hat{\tilde{\iota}}_{1}^{T}, \cdots, \hat{\tilde{\iota}}_{n}^{T}\right]^{T}, \text { where } \hat{\tilde{\iota}}_{r}=\left[\hat{\tilde{\iota}}_{r}^{1}, \cdots, \hat{\tilde{\iota}}_{r}^{m}\right]^{T}, \\
\tilde{\iota}_{u} & =\left[\tilde{\iota}_{u}^{1}, \cdots, \tilde{\iota}_{u}^{m}\right]^{T}, \\
\bar{\iota}_{o} & =\left[\begin{array}{c}
1 \\
\iota_{o}
\end{array}, \cdots, \tilde{\iota}_{o}^{m}\right]^{T}, \\
C & =e_{n} \otimes I_{m}, \\
C_{v} & =\left[\begin{array}{c}
0_{1 \times(n-1)} \\
I_{n-1}
\end{array}\right] \otimes e_{m}, \\
v & =\left[d_{12, G F}, \cdots, d_{1 n, G F}\right]^{T},
\end{aligned}
$$

where $I, e$ and 0 represent the identity matrix, the vector of ones and the matrix of zeros, respectively, with the subscripts denoting their sizes. $\hat{\tilde{\iota}}_{r}^{s}$ represents the estimated biased ionospheric delays from the network processing for receiver $r$ and satellite $s$, and $\bar{\iota}_{o}^{s}$ is the unknown spacial mean of the term $\bar{l}_{r}^{s}$ for all the network stations and the satellite $s$, where the term $\bar{l}_{r}^{s}$ at $t_{i}$ is formulated as:

$$
\bar{\iota}_{r}^{s}\left(t_{i}\right)=\iota_{r}^{s}\left(t_{i}\right)+d_{1, G F}\left(t_{1}\right)-d_{, G F}^{s}\left(t_{1}\right) .
$$

The inverse of the ionospheric variance matrix $S$ (see Eq. A.1) is taken as the weight matrix $W$ for the interpolation, where $S$ is computed as:

$$
S=c_{\iota}^{2}\left[\begin{array}{ll}
R_{11} & R_{12} \\
R_{12}^{T} & R_{22}
\end{array}\right] \otimes C_{s}
$$

with

$$
\begin{aligned}
{\left[\begin{array}{ll}
R_{11} & R_{12} \\
R_{12}^{T} & R_{22}
\end{array}\right] } & =\left[r_{i j}\right], \quad i, j=1, \cdots, n, u \\
C_{s} & =\operatorname{diag}\left(c_{1}^{2}, \cdots, c_{m}^{2}\right)
\end{aligned}
$$

where diag(.) denotes the diagonal matrix of a vector. $c_{\iota}^{s}$ characterizes the overall uncertainty of the ionospheric delays and will be eliminated in Eq. A.14. $c_{s}^{2}$ characterizes the satellite-specific dependency of the signals, which changes with the elevation angle $\beta_{E}^{s}$ of the satellite $s$ :

$$
c_{s}^{2}=\frac{1}{\sin ^{2} \beta_{E}^{s}} .
$$

The spacial correlation function $r_{i j}$ increases with the decreasing between-antenna distance $l_{i j}$ as:

$$
r_{i j}=\exp \left(-\left(\frac{l_{i j}}{l_{o}}\right)^{2}\right)
$$


where $l_{o}$ is a pre-defined applicable inter-station distance for ionospheric spacial correlation, and $\exp ($.$) denotes the natural exponential function. The BLUP of \tilde{\iota}_{u}$ can then be determined as (see Eq. A.1):

$$
\hat{\tilde{\iota}}_{u}=\hat{\bar{\iota}}_{o}+S_{12}^{T} S_{11}^{-1}\left(\hat{\tilde{\iota}}-C \hat{\bar{\iota}}_{o}-C_{v} \hat{v}\right)
$$

with $\hat{\bar{\iota}}_{o}$ and $\hat{v}$ solved from

$$
\left[\begin{array}{ll}
C^{T} S_{11}^{-1} C & C^{T} S_{11}^{-1} C_{v} \\
C_{v}^{T} S_{11}^{-1} C & C_{v}^{T} S_{11}^{-1} C_{v}
\end{array}\right]\left[\begin{array}{c}
\hat{\iota}_{o} \\
\hat{v}
\end{array}\right]=\left[\begin{array}{l}
C^{T} S_{11}^{-1} \tilde{\imath} \\
C_{v}^{T} S_{11}^{-1} \hat{\tilde{\iota}}
\end{array}\right]
$$

\section{References}

Allan, D.W., 1987. Time and frequency (time-domain) characterization, estimation, and prediction of precision clocks and oscillators. IEEE Trans Ultrason Ferroelectrics Freq Control 34(6):647-654. doi:10.1109/T-UFFC.1987.26997.

Baarda, W., 1981. S-transformations and criterion matrices. In: Publications on geodesy, vol. 5, no. 1, second revised edition, Netherlands Geodetic Commission, Delft, the Netherlands, ISBN: 9061322189.

Banville, S., Collins, P., Zhang, W., et al., 2014. Global and regional ionospheric corrections for faster PPP convergence. Navigation 61(2):115-124. doi:10.1002/ navi.57.

Barnes, J.A., Chi, A.R., Cutler, L.S., et al., 1971. Characterization of frequency stability. IEEE Trans Instrum Meas 20(2):105-120.

Bröderbauer, V., Weber, R., 2003. Results of Modelling GPS Satellite Clocks. Österreichische Zeitschrift für Vermessung und Geoinformation, 91(1):38-47.

Collins, P., 2008. Isolating and estimating undifferenced GPS integer ambiguities. In: Proceedings of Inst Navigation National Tech Meet 28-30 January, San Diego, CA, USA, January 2008, pp. 720-732.

Dai, L., Wang, J., Rizos, C., et al., 2003. Predicting atmospheric biases for realtime ambiguity resolution in GPS/GLONASS reference station networks. J Geod 76(11-12):617-628. doi:10.1007/s00190-002-0286-1.

Dow, J.M., Neilan, R.E., Rizos, C., 2009. The International GNSS Service in a changing landscape of Global Navigation Satellite Systems. J Geod 83(3-4):191198. doi:10.1007/s00190-008-0300-3. 
El-Mowafy, A., 2012. Precise Real-Time Positioning Using Network RTK, Chapter 7, Global Navigation Satellite Systems: Signal, Theory and Applications. Edited by Shuanggen Jin, Publisher: InTech. doi:10.5772/1134.

El-Mowafy, A., Al-Musawa, M., 2009. Machine Automation using RTK GPS Positioning. In Proc ISMA09, March 23-26, 2009. doi:0.1109/ISMA.2009.5164849.

El-Mowafy, A., Deo, M., Kubo, N., 2016. Maintaining real-time precise point positioning during outages of orbit and clock corrections. GPS Solut, published online, 19 November 2016. doi:10.1007/s10291-016-0583-4.

Ge, M., Gendt, G., Rothacher, M., et al., 2008. Resolution of GPS carrier-phase ambiguities in precise point positioning (PPP) with daily observations. J Geod 82(7):389-399. doi:10.1007/s00190-007-0187-4.

Geng, J., Meng, X., Dodson, A.H., et al., 2010. Rapid re-convergences to ambiguityfixed solutions in precise point positioning. J Geod 84(12):705-714. doi:10.1007/ s00190-010-0404-4.

Geoscience Australia, 2017. Data from the Geoscience Australia.

Goldberger, A.S., 1962. Best linear unbiased prediction in the generalized linear regression model. Journal of the American Statistical Association 57(298):369375 .

Greenhall, C.A., 2001. Kalman Plus Weights: A Time Scale Algorithm. In Proc PTTI 2001, Long Beach, California, November 2001, pp. 445-454.

Griffiths, J., Ray, J.R., 2009. On the precision and accuracy of IGS orbits. J Geod 83(3):277-287. doi:10.1007/s00190-008-0237-6.

Hauschild, A., Montenbruck, O., 2009. Kalman-filter-based GPS clock estimation for near real-time positioning. GPS Solut 13(3): 173-182. doi:10.1007/ s10291-008-0110-3.

Herring, T.A., Davis, J.L., Shapiro, I.I., 1990. Geodesy by radio interferometry: The application of Kalman filtering to the analysis of very long baseline interferometry data. J Geophys Res 95(B8):12561-12581. doi:10.1029/JB095iB08p12561.

Hofmann-Wellenhof, B., Lichtenegger, H., Wasle, E., 2008. GNSS-Global Navigation Satellite Systems: GPS, GLONASS, Galileo, and more. Springer-Verlag Wien. doi: 10.1007/978-3-211-73017-1. 
Huang, G.W., Zhang, Q., Xu, G.C., 2014. Real-time clock offset prediction with an improved model. GPS Solut 18(1):95-104. doi:10.1007/s10291-013-0313-0.

IGS clock, 2017. International GNSS Service, GNSS Final Combined Satellite and Receiver Clock Solution (30 second) Product, Greenbelt, MD, USA:NASA Crustal Dynamics Data Information System (CDDIS), Accessed on January 31, 2017 at doi:10.5067/GNSS/gnss_igsclk30_001.

IGS orbit, 2017. International GNSS Service, GNSS Final Combined Orbit Solution Product, Greenbelt, MD, USA:NASA Crustal Dynamics Data Information System (CDDIS), Accessed on January 31, 2017 at doi:10.5067/gnss/gnss_igsorb_001.

Janssen, V., Haasdyk, J., 2011. Assessment of Network RTK Performance using CORSnet-NSW. In Proceedings of IGNSS 2011 Symposium, 15-17 November 2011, Sydney, Australia.

Khodabandeh, A., Teunissen, P., 2015. An analytical study of PPP-RTK corrections: precision, correlation and user-impact. J Geod 89 (11): 1109-1132. doi:10.1007/ s00190-015-0838-9.

Kleusberg, A., Teunissen, P.J.G., 1996. GPS for geodesy. Springer-Verlag, Berlin, Heidelberg. doi:10.1007/BFb0117676.

Krawinkel, T., Schön, S., 2016. Benefits of receiver clock modeling in code-based GNSS navigation. GPS Solut 20(4):687-701. doi:10.1007/s10291-015-0480-2.

Laurichesse, D., 2011. The CNES Real-time PPP with undifferenced integer ambiguity resolution demonstrator. In Proc ION GNSS 2011, Portland, OR, September 2011, pp. 654-662.

Laurichesse, D., Mercier, F., Berthias, J.P., 2010. Real-time PPP with Undifferenced Integer Ambiguity Resolution, Experimental Results. In Proc ION GNSS 2010, Portland, OR, September 2010, pp. 2534-2544.

Leandro, R., Landau, H., Nitschke, M., et al., 2011. RTX Positioning: The Next Generation of cm-accurate Real-time GNSS Positioning. In Proc ION GNSS 2011, Portland, OR, September 2011, pp. 1460-1475.

Mervart, L., Lukes, Z., Rocken, C., et al., 2008. Precise Point Positioning with ambiguity resolution in real-time. In Proc ION GNSS 2008, Savannah, GA, September 2008, pp. 397-405. 
Moritz, H., 1978. Least-squares collocation. Reviews of Geophysics 16(3):421-430. doi:10.1029/RG016i003p00421.

Noll, C.E., 2010. The Crustal Dynamics Data Information System: A resource to support scientific analysis using space geodesy. Adv Space Res 45(12):1421-1440, ISSN 0273-1177. doi:10.1016/j.asr.2010.01.018.

Odijk, D., 2002. Fast precise GPS positioning in the presence of ionospheric delays. Doctoral thesis, Delft University of Technology, ISBN:90-804147-2-7.

Odijk, D., Verhagen, S., 2007. Recursive Detection, Identification and Adaptation of Model Errors for Reliable High-Precision GNSS Positioning and Attitude Determination. In: Proceedings of the 3rd International Conference on Recent Advances in Space Technologies, June 2007, doi:10.1109/RAST.2007.4284068.

Odijk, D., Khodabandeh, A., Nadarajah, N., et al., 2017. PPP-RTK by means of S-system theory: Australian network and user demonstration. Journal of Spatial Science 62(1): 3-27. doi:10.1080/14498596.2016.1261373.

Odijk, D., Zhang, B., Khodabandeh, A., et al., 2016. On the estimability of parameters in undifferenced, uncombined GNSS network and PPP-RTK user models by means of S-system theory. J Geod 90(1):15-44. doi:10.1007/s00190-015-0854-9.

Pratt, J., Axelrad, P., Larson, K.M., et al., 2013. Satellite clock bias estimation for iGPS. GPS Solut 17(3):381-389. doi:10.1007/s10291-012-0286-4.

Qu, M., 2012. Experimental studies of wireless communication and GNSS kinematic positioning performance in high-mobility vehicle environments. Master thesis, Faculty of Science and Engineering, Queensland University of Technology, March 2012.

Riley, W.J., 2008. Handbook of Frequency Stability Analysis. NIST Special Publication 1065, July 2008.

RTCM Standard 10403.2, 2013. RCTM Standard 10403.2, differential GNSS (Global Navigation Satellite Systems) Services - Version 3, November 7, 2013.

Senior, K.L., Ray, J.R., Beard, R.L., 2008. Characterization of periodic variations in the GPS satellite clocks. GPS Solut 12(3):211-225. doi:10.1007/s10291-008-0089-9.

Teunissen, P.J.G., 1985. Zero Order Design: Generalized inverses, adjustment, the datum problem and S-transformations. In: Optimization and Design of Geodetic Networks. Springer-Verlag, Berlin, Heidelberg, pp. 11-55. doi:10.1007/ 978-3-642-70659-2_3. 
Teunissen, P.J.G., 1990. Quality control in integrated navigation systems. In: IEEE Aerospace and Electronic Systems Magazine, 5(7):35-41. doi:10.1109/PLANS. 1990.66172 .

Teunissen, P.J.G., Khodabandeh, A., 2013. BLUE, BLUP and the Kalman filter: some new results. J Geodesy 87(5):461-473. doi:10.1007/s00190-013-0623-6.

Teunissen, P.J.G., Khodabandeh, A., 2015. Review and principles of PPP-RTK methods. J Geod 89(3): 217-240. doi:10.1007/s00190-014-0771-3.

Teunissen, P.J.G., Odijk, D., Zhang, B., 2010. PPP-RTK: Results of CORS NetworkBased PPP with Integer Ambiguity Resolution. J Aeronaut, Astronaut Aviat, Series A, 42(4):223-230.

van Dierendonck, A.J., McGraw, J.B., Brown, R.G., 1984. Relationship between Allan variances and Kalman filter parameters. In Proc PTTI 1984, Greenbelt, MD, 1984, pp. 273-293.

Vollath, U., Talbot, N.C., Glocker, M., et al., 2012. GNSS signal processing with delta phase, Patent Pub. No.: WO2012151006 A1, Trimble Navigation Limited, November 2012.

Weber, G., Dettmering, D., Gebhard, H., 2005. Networked Transport of RTCM via Internet Protocol (NTRIP). In: Sansò F. (eds) A Window on the Future of Geodesy. IAG Symp., 128, pp. 60-64, Springer, Berlin, Heidelberg. doi:10.1007/ 3-540-27432-4_11.

Wübbena, G., Schmitz, M., Bagge, A., 2005. PPP-RTK: precise point positioning using state-space representation in RTK networks. In Proc ION GNSS 2005, Long Beach, CA, September 2005, pp. 2584-2594.

Yu, X., Gao, J., 2017. Kinematic Precise Point Positioning Using Multi-Constellation Global Navigation Satellite System (GNSS) Observations. ISPRS Int J Geo-Inf 2017, 6(1), 6. doi:10.3390/ijgi6010006.

Zumberge, J.F., Heflin, M.B., Jefferson, D.C., et al., 1997. Precise point positioning for the efficient and robust analysis of GPS data from large networks. J Geophys Res 102(B3):5005-5017. doi:10.1029/96JB03860. 